\title{
Quantum electrodynamics in modern optics and photonics: tutorial
}

\author{
David L. Andrews ${ }_{g}{ }^{1, *}$ (i) David S. Bradshaw ${ }^{1}$ Kayn A. Forbes $_{9}{ }^{1}$ (i) and A. Salam ${ }^{2,3,4}$ (i) \\ ${ }^{1}$ School of Chemistry, University of East Anglia, Norwich Research Park, Norwich NR4 7TJ, UK \\ ${ }^{2}$ Department of Chemistry, Wake Forest University, Winston-Salem, North Carolina 27109-7486, USA \\ ${ }^{3}$ Physikalische Institut, Albert-Ludwigs-Universität-Freiburg, Hermann-Herder-Strasse 3, D-79104, Freiburg, Germany \\ ${ }^{4}$ Freiburg Institute for Advanced Studies (FRIAS), Albertstrasse 19, D-79104, Freiburg, Germany \\ *Corresponding author: d.I.andrews@uea.ac.uk
}

Received 15 November 2019; revised 19 February 2020; accepted 20 February 2020; posted 21 February 2020 (Doc. ID 383446); published 19 March 2020

\begin{abstract}
One of the key frameworks for developing the theory of light-matter interactions in modern optics and photonics is quantum electrodynamics (QED). Contrasting with semiclassical theory, which depicts electromagnetic radiation as a classical wave, QED representations of quantized light fully embrace the concept of the photon. This tutorial review is a broad guide to cutting-edge applications of QED, providing an outline of its underlying foundation and an examination of its role in photon science. Alongside the full quantum methods, it is shown how significant distinctions can be drawn when compared to semiclassical approaches. Clear advantages in outcome arise in the predictive capacity and physical insights afforded by QED methods, which favors its adoption over other formulations of radiation-matter interaction. () 2020 Optical Society of America
\end{abstract}

https://doi.org/10.1364/JOSAB.383446

\section{INTRODUCTION}

Formulations of theory represent the foundation for describing and interpreting all forms of optical interaction with matter. As such, they not only represent the basis for technical and quantitative descriptions; they also offer frameworks for conceiving and understanding the nature of such interactions, and their underlying mechanisms. In a wide-ranging field of applications in optics and photonics-including the science and technology of optical materials, spectroscopic analysis, optical sensors, laser frequency conversion, nanoscience, photophysics, photochemistry, and photobiology—one may observe that two essentially different kinds of theory are commonly applied.

The most prevalent is semiclassical theory (SCT), a framework in which light is treated classically, usually as a sinusoidal wave, and matter is treated by methods based on quantum mechanics [1]. Such a formulation formally entails classical electrodynamics based on wave optics, as it has been taught for well over a century. Sustained by its remarkably broad applicability, SCT has found wide acceptance in the successive generations of textbooks from which practitioners usually learn the basics, with the tacit assumption that it is easier to grasp than full quantum theories. Indeed, much of the theory in physical optics is almost entirely classical, in the sense that material parameters such as bulk optical susceptibilities are treated phenomenologically, in terms of scalar or tensor parameters whose mathematical constructs are not always of direct concern. However, it would have to be acknowledged that the correct form and quantitative behavior of those parameters are ultimately only derivable from quantum mechanical representations. Of course, even the band structures of bulk materials can be interpreted in no other way-but these primarily concern materials, not light. Despite its shortcomings, an SCT formulation of optics can still be considered serviceable.

The other mainstream representation of theory for optical interactions is quantum electrodynamics (QED) [2-10]. Here, both matter and radiation are treated with the full rigor of quantum mechanics, and as such they naturally express processes and interactions in terms of light quanta, i.e., photons [11]. In the sphere of optics, this cast of theory is commonly applied in a formulation that treats space and time nonrelativistically, since none of the salient charges move at anything approaching the speed of light. Just as with SCT, this framework too is based on Maxwell's equations for the electromagnetic fields, but here, those fields are promoted from regular variables to acquire the status of quantum operators, which act on radiation field state vectors. In consequence, quantum principles are consistently applied to the entirety of each and every system, whether or not charges or photons are present. In particular, for applications in optics, this version of theory intrinsically subsumes both quantum optics and quantum mechanics.

It is significant that, while the notion of photons is invariably deployed at some stage in describing even the simplest optical processes, such as the electronic transitions that result from 
absorption-even in SCT-the photon is strictly a concept that is at odds with the assumptions of that theory. The Planck relation between energy and frequency is clearly not a construct that can be elicited from any classical wave representation. Nonetheless, conventional SCT treatments of absorption and emission afford neat, tractable introductions to the applications of time-dependent perturbation theory [12-14], while the development of higher-order responses leads gently into nonlinear optics [15-17]. As such, this branch of theory has become an almost incidental assumption, frequently sustained in the descriptions of experimental phenomena, even at the research level.

One of the most important reasons for the sustained popularity of SCT is that, when it is allowed the logically inconsistent deployment of the Planck relation in judicious places, it often provides results that are in correct agreement with experiment, and with QED, to the level of experimental error. Yet SCT is flawed at a fundamental level —not just because the world of light and matter is, in fact, comprehensively quantum mechanical, but for several other reasons. Where failures of SCT arise, they are found not only in some obvious departure of agreement with experiment in relatively exotic or high-precision physics, where QED has scored some of its most celebrated successes (calculations of the fine-structure constant, relativistic effects in heavy atoms, and fine-structure level splitting afford significant examples [18-20]). Despite notable attempts by Jaynes and co-workers [21,22], it proves impossible to elicit a correct representation of spontaneous emission [5,23], the generic term for all familiar forms of fluorescence, phosphorescence, and luminescence. This is the most striking and ultimately unacceptable manifestation of a fundamental flaw in SCT.

In SCT, any electronic excited state secured as a solution of the time-independent Schrödinger equation is necessarily a stable, stationary state: there is no system operator to act as a perturbation and provide for state decay, unless, of course, light of the appropriate wavelength is present, as in the special case of stimulated emission. Thus, isolated excited atoms might incorrectly be expected to have an infinite excited state lifetime, precisely because they are stationary eigenstates of the atomic Hamiltonian. Under SCT, the electric field of radiation $\boldsymbol{e}$ is zero when no light is present, whereas in QED, a multipolar coupling operator such as $\boldsymbol{- \mu} \cdot \boldsymbol{e}$ representing an electric dipole (E1) $\boldsymbol{\mu}$ engaging with an electric field vector $\boldsymbol{e}$ is never zero, because $\boldsymbol{\mu}$ and $\boldsymbol{e}$ are both operators, one acting on the states of the matter and the other on the radiation field. The same applies when this dipole coupling is more formally expressed (as we do later) in terms of the electric displacement field. Moreover, the system Hamiltonian in QED comprises not only terms for the matter and multipolar (or other) coupling to radiation, but also a radiation Hamiltonian that is always present $[5,8]$. Consistent with the finite ground state of every quantum mechanical harmonic oscillator, the physical corollary is that vacuum fluctuations perturb every material excited state, providing a mechanism for decay transitions to occur.

Terms such as "vacuum fluctuations" or "vacuum field" might, incidentally, be considered unfortunate, being suggestive of exotic phenomena that can be identified only in regions of space devoid of matter, whereas they are, in fact, universally present [24]. However, they represent only one form of physical interpretation of the relatively simple mathematics; it is not entirely necessary to deploy such a viewpoint to attain the correct QED form of spontaneous emission. Loose terminology is always a problem: as a counterargument, it could be said that several terms more widely used in the semiclassical literature can also be misleading, such as the common description of an absorber as an "oscillator" or a "dipole," without identifying the key distinction between static and transition forms of oscillation and dipole.

There are two widely held misconceptions concerning QED. One is a common perception that the subject is substantially more difficult and complicated to apply than SCT. In fact, it is easy to demonstrate that, in describing common optical processes, QED is no more difficult than SCT: the latter is scarcely any simpler even when the complexities and assumptions of classical wave theory are hidden away, as is often the case in textbook treatments. A second objection is that (despite its unique success with spontaneous emission) the application of QED is required only for high-precision calculations [25]. Both the premise and conclusion of such logic are, of course, spurious; the success of QED in calculating the fine-structure constant and other such quantities, with unmatched precision, serves to underscore its complete scientific validity.

An unwarranted and unnecessary gulf thus seems to have become established, principally for historical reasons, between the fully quantized formalisms of quantum optics, and the largely semiclassical treatments of atomic and molecular interactions with light. It is legitimate to pose the question of why one should choose to deploy kinds of theory that do not correctly reflect the quantum nature of light, for the problems and faults of SCT presented above are not its only deficiencies. At a time when the whole sphere of technology is being transformed by engineered photonics (a field commonly called "quantum technologies"), and science has moved into what has already been dubbed the "century of the photon," the attraction of framing theory in a formulation that duly represents the quantum nature of electromagnetic radiation is increasingly evident. The photon concept is clearly a requisite for understanding and correctly representing operations in quantum technology. While SCT often provides extremely similar results to QED for systems with large quantum numbers, it is important to bear in mind that any such notion of large numbers makes sense only with regard to a specified interaction volume, a few implications of which are discussed in Appendix A.

A comparison of QED and SCT is widely available in both textbooks, such as Ref. [1], and the literature, including the in-depth review article by Milonni [26]. Without heavy repetition of the earlier work, this tutorial review aims to objectively illustrate not only applications-primarily in the sphere of condensed phase optics and photonics - but also insights into mechanisms afforded by a photon-based perspective. Several recent examples are drawn from the fields of optical manipulation and structured light. While the nature of the subject is of course mathematical, and demands the language of mathematics, our intention is to keep equations to a minimum, in order to focus on physical and interpretive aspects. The heightened predictive power of QED is also exhibited in areas where there are evident shortcomings in theory cast in a classical or semiclassical guise. 


\section{BASIC FORMULATIONS}

To address the mathematical detail, it will be helpful to begin with a summary of key differences in the foundational equations of QED and SCT. The most substantial difference between these theories is encapsulated in their distinct forms of system Hamiltonian, exemplified in the following analysis. Here, we shall use the term "molecule" in a generic sense, to signify any material entity that is electrically neutral, and which has an identifiable electronic integrity. As such, the description in principle applies to free atoms, molecules, chromophore groups within molecules, and even larger systems with extended quantum behavior such as "quantum dots." With care, application can also be made to guest species in a host lattice-notably, rare-earth dopants in crystal media with significantly different absorption features.

First, we consider the optical interactions of any such single "molecule." The SCT and counterpart QED system Hamiltonians are cast as

$$
\begin{gathered}
H_{\text {sys }}^{\mathrm{SCT}}=H_{\mathrm{mol}}+H_{\mathrm{int}} ; \\
H_{\text {sys }}^{\mathrm{QED}}=H_{\mathrm{mol}}+H_{\mathrm{rad}}+H_{\mathrm{int}} .
\end{gathered}
$$

Here, in both equations, $H_{\text {mol }}$ represents the molecular Hamiltonian, while in the second expression, $H_{\mathrm{rad}}$ represents a Hamiltonian for the radiation field. In both cases, the full Hamiltonian is expressible in the form $H=H_{0}+H_{\text {int }}$, where the interaction Hamiltonian term provides for perturbations that allow transitions within a basis set of states, which are the eigenstates of $H_{0}$. Nonetheless, a difference of meaning in $H_{0}$ in each of these interpretations signifies that the corresponding eigenstates take a significantly different form in the two theories.

In the case of SCT, the basis states are simply eigenstates of $H_{\text {mol }}$, expressible in the form $|\mathrm{mol}\rangle=\left|E_{m}^{\xi}\right\rangle$ for a molecule $\xi$ in its $m$ th electronic state: additional labels may be inserted within the ket, as necessary, to specify other internal degrees of freedom. Formally, these quantum states are formulated in a Hilbert space. However, with $H_{\text {rad }}$ included in the treatment from the outset in QED theory [5,8], the unperturbed Hamiltonian $H_{0}$ comprises the sum of $H_{\text {mol }}$ and $H_{\text {rad }}$. This means that the basis states employed in this construct are separable molecule-field product states, given in general by $|\mathrm{mol}\rangle|\mathrm{rad}\rangle=|\mathrm{mol} ; \mathrm{rad}\rangle=\left|E_{m}^{\xi} ; n(\boldsymbol{k}, \eta)\right\rangle$, i.e., products of Hilbert states for the material and Fock states for the radiation. Here, number state representations are used to specify the electromagnetic field, $n(\boldsymbol{k}, \eta)$, with $n$ designating the number of photons of wave vector $\boldsymbol{k}$ and polarization index $\eta$ : the latter two variables together designate a plane-wave photon mode. While the radiation field may be described in other ways, for example as a coherent state, the number state approach usually enables a more direct connection to the light quanta: it also has the advantage of being the simplest basis, in terms of which any other can in principle be expressed. For example, variations in $\boldsymbol{k}$ vector are accommodated in the quantum description of structured light (a point we shall return to in Section 5). Notably, in the QED formulation, $H_{\text {int }}$ is an operator in the space of both matter and radiation states, and therefore it is always part of the system Hamiltonian, whereas it only features in SCT if electromagnetic radiation is present.

By extension to an assembly of molecules, individually distinguished by a label $\xi$, the QED multipolar Hamiltonian takes a particularly simple form, when deploying the standard, PowerZienau-Woolley (PZW) formulation [9,27-29],

$$
H_{\text {sys }}^{\mathrm{QED}}=\sum_{\xi} H_{\mathrm{mol}}+H_{\mathrm{rad}}+\sum_{\xi} H_{\mathrm{int}} .
$$

This equation deserves a number of comments before we go further. It is striking that, while QED was first formulated to tackle the interactions of fundamental charges and photons, the subsequent development of molecular QED wrought this beautiful simplicity to the interactions of larger, electrically neutral species-interactions both with light, and among themselves. Notably, there is no direct interaction between "molecules" in the exact multipolar form of system Hamiltonian, as is evident from the lack of any double-sum in the structure of Eq. (3). In this formalism, intermolecular interactions operate only through mediation of the electromagnetic field, which in the quantum formulation has to mean photons (whether real or virtual, with the latter being unobserved).

To be clear, the lack of any intermolecular terms in Eq. (3) relating to direct Coulombic interactions formally signifies that such static or "longitudinal" fields cancel out exactly, in the detailed multipolar form [3]. All forms of electrodynamic coupling between molecules are necessarily mediated by the exchange of photons $[5,8,9]$. (The differences that arise in the "minimal-coupling" formulation are well documented in these three references and elsewhere: they are not relevant to the comparisons with SCT, and they lie beyond the scope of the present review.) This does not mean, however, that the effects of static fields cannot be accommodated in the theory. In fact, while externally applied static electric and magnetic fields can be treated as additional zero-frequency perturbations-see, for example, Refs. [30,31] — their effect can also be correctly identified as the result of virtual photon coupling with static multipoles of the corresponding electric or magnetic kind. Adopting this form of coupling enables proper account of the influence of local molecular dipoles and surface potentials, as for example in a recent study of the fluorescence energy transfer at membrane surfaces [32].

The requirements for using a molecular QED formulation are principally that the component particles are slow-moving, and electronically distinct. These constraints preclude only direct application (without due modifications being implemented) to particles moving at relativistic speeds, or those with wave function overlap leading to exchange integral energies. Somewhat misleadingly, the term "dilute gas" is sometimes deployed to signify insignificant overlap between the wave functions of component particles. In practice, the notion of distinct electronic integrity suffices, enabling perfectly sound applications to be made to real gases, and to most liquids and solutions. By interpreting the "molecular" Hamiltonian appropriately, it is entirely possible to take into account both electronic and nuclear motions, most commonly through a Born-Oppenheimer separation of intramolecular vibrations. Hence, for example, many 
studies of the molecular Raman effect have been pursued using QED methods; see, for example, Refs. [5,33-38].

With limitations, molecular solids are also valid objects of such theory: here, the constraint on direct applicability simply means nonconductivity, and an exclusion of delocalized phonon or polariton excitations. By a logical extension, the formulation of molecular QED can be further applied to regular dielectric solids, for which the elementary components may be regarded as comprising unit cells [39,40]. Indeed, the foundations of "classical" nonlinear optical susceptibility are based on an exactly similar premise of dependence on atom density in most classic texts; see, for example, Refs. [17,41,42]. Nonetheless, there is a caveat here, for it is evident that departures from the simplicity of a conventional molecular QED formulation must start to be apparent whenever coupling occurs between the intraparticle eigenstates of $H_{\mathrm{mol}}$ and delocalized states of the condensed phase bulk. Such coupling can engender thermalization and dissipation processes, some most obviously manifest as damping [43] — a topic we shall shortly return to in another connection.

Returning again to Eq. (3), we now focus on the middle term. In the second quantized representation, the Hamiltonian for the radiation field may be written as

$$
H_{\mathrm{rad}}=\sum_{\boldsymbol{k}, \eta}\left\{a^{\dagger(\eta)}(\boldsymbol{k}) a^{(\eta)}(\boldsymbol{k})+\frac{1}{2}\right\} \hbar c k,
$$

and described as a collection of harmonic mode oscillators with circular frequency $\omega=c k$. The noncommuting operators $a^{(\eta)}(\boldsymbol{k})$ and $a^{\dagger(\eta)}(\boldsymbol{k})$ are lowering (annihilation) and raising (creation) operators, respectively. In the case of the number states, which are exact eigenstates of the radiation Hamiltonian, these operators serve to decrease or increase by one the number of photons of mode $(\boldsymbol{k}, \eta)$ in the radiation field, via $a^{(\eta)}(\boldsymbol{k})|n(\boldsymbol{k}, \eta)\rangle=n^{1 / 2}|(n-1)(\boldsymbol{k}, \eta)\rangle$ and $a^{\dagger(\eta)}(\boldsymbol{k})|n(\boldsymbol{k}, \eta)\rangle=(n+1)^{1 / 2}|(n+1)(\boldsymbol{k}, \eta)\rangle$. The ordered product $a^{\dagger(\eta)}(\boldsymbol{k}) a^{(\eta)}(\boldsymbol{k})$ is called the number operator, $n(\boldsymbol{k}, \eta)$ signifying an integer number of photons. For some types of laser beam, to correctly represent a number that is subject to fluctuation and aspects of photon statistics, it is expedient to employ other forms of radiation state containing a suitable superposition, typically, the previously mentioned coherent states. From Eq. (4), it is apparent that the entire energy of the radiation field is identical to that of the populated subset of an infinite set of quantum harmonic oscillators, $H_{\text {rad }}=(n+1 / 2) \hbar \omega$, $n=0,1,2, \ldots$, where the second term denotes the zero point energy of the vacuum, meaning $n=0$, i.e., an absence of photons.

Before proceeding further, it is interesting to observe an immediate conclusion that can be drawn from a comparison between the forms of Eqs. (1) and (2). The radiation Hamiltonian, $H_{\text {rad }}$, of Eq. (4) does not commute with $H_{\text {int }}$, whose leading terms have a linear dependence on the photon creation and annihilation operators. (As we shall see, each individual engagement of the electric or magnetic field operators necessitates the involvement of one or other of these boson operators.) Consequently, stationary states of $H^{\text {SCT }}$ cannot be stationary states of the complete $H^{\mathrm{QED}}$. This, in a nutshell, is why SCT ultimately fails to correctly account for spontaneous emission, among much else. In fact, since the photon creation operator acts on the radiation vacuum state to give a nonzero result-as follows from the equations above with $n=0$-it is a simple matter, using methods to be detailed in the next section, to derive a correct expression for the rate of spontaneous emission,

$$
\langle\Gamma\rangle=\frac{\omega^{3}}{3 \pi \varepsilon_{0} \hbar c^{3}}\left|\boldsymbol{\mu}^{m 0}\right|^{2} .
$$

The above result, a textbook example [5], represents the rate for an isotropic source undergoing an E1 allowed transition, from the $m$ th excited state down to the ground state, subject to energy conservation $E_{m 0} \approx \hbar \omega$, where $\omega=c k$ is the circular frequency. The result, Eq. (5), can be easily extended to incorporate the influence of a dielectric medium [39,40].

In the next section, we outline the formal components for a QED development of theory, providing the formal basis for both simple and substantially more intricate forms of optical interaction. This establishes the rigorous basis for such a development; it will then be unnecessary to rehearse the entire basis in each and every implementation.

\section{DEVELOPING QED THEORY FOR APPLICATIONS TO SPECIFIC OPTICAL PROCESSES}

It is not unreasonable to assert that every formulation of theory in the realm of optics has, at its roots, one or more of Maxwell's equations. As observed earlier, this is just as true for SCT as for QED; the key difference is that, in the latter, the fields appearing in those equations are promoted to operator status. Since Maxwell's work represents an accepted common ground, we shall begin the following development at a higher level: interested readers may find the underlying development, beginning with Maxwell's equations, in the standard textbooks $[1,5,8,44-47]$.

\section{A. Field Expansions}

To elicit the mechanistic form of optical interactions, it is necessary to represent the fundamental nature of the engagement between light and matter. Once again, there is a widely accepted representation (though, in this case, an understood acknowledgement that it is inexact): the E1 approximation. Physically, this may be argued on the basis that, at least for dielectric materials, the electric field of any optical radiation will be the dominant influence, acting upon local charge distributions to produce shifts in the equilibrium centers of positive and negative charge. Although this E1 approximation does not have to be applied either in QED or in SCT, light-molecule interactions mediated by an E1 are much more efficient than those for an electric quadrupole (E2), any other higher-order electric multipole, or even a magnetic dipole (M1) —although both M1 and E2 forms of coupling become important in studies on chirality [48-51]. Therefore, for ease of explanation, we will restrict ourselves to the E1 approximation. In this case, the interaction (PZW) Hamiltonian is expressible as $[5,8]$

$$
H_{\mathrm{int}}=-\varepsilon_{0}^{-1} \mu_{i}(\xi) d_{i}^{\perp},
$$


where $\mu_{i}(\xi)$ is the electric-dipole moment operator. The Latin subscript denotes a Cartesian component with an implied summation convention for repeating indices. Moreover, $d_{i}^{\perp}$ is a component of the transverse electric displacement operator for a plane wave, expressible (in its Fourier mode expansion form) as

$$
\begin{aligned}
d_{i}^{\perp}(\boldsymbol{r})= & i \sum_{\boldsymbol{k}, \eta}\left(\frac{\hbar c k \varepsilon_{0}}{2 V}\right)^{\frac{1}{2}} \\
& \times\left\{e_{i}^{(\eta)}(\boldsymbol{k}) a^{(\eta)}(\boldsymbol{k}) \mathrm{e}^{i \boldsymbol{k} \cdot \boldsymbol{r}}-\bar{e}_{i}^{(\eta)}(\boldsymbol{k}) a^{\dagger(\eta)}(\boldsymbol{k}) \mathrm{e}^{-i \boldsymbol{k} \cdot \boldsymbol{r}}\right\} .
\end{aligned}
$$

Here, $e_{i}^{(\eta)}(\boldsymbol{k})$ is the electric polarization unit vector with the complex conjugate variable denoted by an overbar, $\boldsymbol{r}$ is an arbitrary position in space, and $V$ is an arbitrary quantization volume. Here, the linear dependence on photon creation and annihilation operators, alluded to earlier with respect to $H_{\text {int }}$, is clearly obvious. In passing, it is worth noting that the magnetic induction field of the radiation field, signifying the counterpart field in electromagnetic radiation, has an exactly analogous expansion, also linear in $a^{(\eta)}(\boldsymbol{k})$ and $a^{\dagger(\eta)}(\boldsymbol{k})[5,8,10]$. In consequence, every interaction of light has to involve the annihilation or creation of photons - or both. (Where these interactions involve virtual photons, the creation and annihilation always occur as paired events [49]).

\section{B. Perturbation Theory}

A frequently used analytical method for tackling time evolution in all but the simplest complex quantum systems is time-dependent perturbation theory. For SCT, this appears to be a natural consequence of partitioning the Hamiltonian into unperturbed, $H_{0}$, and perturbed parts, according to whether or not light (and hence, in this interpretation, $H_{\text {int }}$ ) is present. In QED, the distinction is equally effective, but its basis is perhaps more subtle, since none of the operators can be identified with zero: as should be the case in any quantum theory, the operators are not to be defined in terms of a specific state of the system. Nonetheless, in the realm of application of both formulations, the coupling between electromagnetic radiation and matter, represented by the interaction Hamiltonian, $H_{\text {int }}$, is associated with energies that are small (due to weak fields) relative to the Coulomb binding interactions within the molecule itself (which arise from a much higher field strength).

Before proceeding with the detail, it is worth observing that in high-field optics, for both SCT and QED, perturbation theory breaks down at high levels of optical intensity. The threshold for departures from perturbation theory is commonly around $10^{17} \mathrm{Wm}^{-2}$; at such intensities, molecular bonds typically begin to break and electrons detach. Under these circumstances, one common approach is Floquet theory, which provides an exact solution to the time-dependent Schrödinger equation for a Hamiltonian that is periodic in time [52]. In an SCT framework it has, for example, been applied to the study of short laser pulses of high intensity interacting with atoms [53], in particular multiphoton resonances, high-harmonic generation, and above-threshold ionization processes. For quantum fields, Floquet theory is less commonly employed, but examples include application to high-order harmonic emission and hyper-Raman spectra [54], and the dynamics of strong field coupling [55]. For a two-level system interacting with a single-mode quantum radiation field, the well-known Jaynes-Cummings model illustrates the intricate convolutions necessary to secure a result using SCT [56]. Much more recently, there has been renewed interest in the dynamical solution to this model, in the context of the breakdown of perturbation theory and the rotating-wave approximation (RWA) at the onset of the ultrastrong coupling regime [57].

Let us return to the more widely adopted perturbation theory methods. By employing the standard techniques of time-dependent perturbation theory, the influence of the perturbation in causing the total system to evolve from some initial state, $|i\rangle$, to some final state, $|f\rangle$, may readily be evaluated. The probability amplitude for the transition $|f\rangle \leftarrow|i\rangle$ is expressible in terms of a transition operator, $T$, whose matrix elements are usually written as $M_{f i}=\langle f|T| i\rangle$. The operator $T$ is itself expanded as a series in powers of the perturbation operator [58,59],

$$
T=T^{(1)}+T^{(2)}+T^{(3)}+T^{(4)}+\ldots,
$$

with

$$
T^{(1)}=H_{\text {int }},
$$

$$
T^{(2)}=H_{\mathrm{int}} \frac{1}{E_{i}-H_{0}+i \varepsilon} H_{\mathrm{int}},
$$

and so forth, where $E_{i}$ is the energy of state $|i\rangle$. Notice again that $H_{0}$ and its eigenenergies differ according to the definition of the system in SCT and QED. The introduction of an infinitesimal quantity $\varepsilon$ ensures analyticity, understanding that it is to be taken in the limit $\varepsilon \rightarrow^{+} 0$. Commonly, line-shape factors associated with damping and dissipative losses are not included in formulations at this level, primarily because they are associated with material components and local fields beyond the compass of the system under study, and commonly associated with temporally stochastic or heterogeneous forms of interaction. As such, these may, for example, include coupled motion in structural vibrations, or the electronic influence of neighboring matter: only in the case of atomic gases at low pressure can it be assumed that radiative decay properly accounts for any experimentally determined linewidth.

It is incorrect to assume that the arbitrary infinitesimal can simply be replaced by any all-encompassing phenomenological damping factor: such factors can be assimilated into the theory, but they are not in general amenable to tractable analytic expression. Any inclusion of a generic phenomenological representation of damping must therefore be essentially a pragmatism, for either SCT or QED. Its introduction can certainly obscure the rigor at the core of the QED formulation, since breaking the symmetry of time-reversal leads unavoidably to a non-Hermitian Hamiltonian [60,61]. The implications are indeed more easily understood from the QED perspective, since to secure real expectation values for the radiation fields-and molecular state energies - the corresponding quantum operators in each respect have to be Hermitian. The eigenfunctions of such operators cannot accommodate beam dissipation or 
Table 1. Four Possibilities for Matrix Element Evaluation $^{a}$

\begin{tabular}{|c|c|c|}
\hline $\begin{array}{l}\downarrow \text { Hilbert Space } \backslash \\
\text { Fock Space } \rightarrow\end{array}$ & $|f\rangle_{\mathrm{rad}}=|i\rangle_{\mathrm{rad}}$ & $|f\rangle_{\mathrm{rad}} \neq|i\rangle_{\mathrm{rad}}$ \\
\hline$|f\rangle_{\mathrm{mol}}=|i\rangle_{\mathrm{mol}}$ & potential energy & $\begin{array}{l}\text { rate of parametric } \\
\text { process, e.g., } S H G\end{array}$ \\
\hline$|f\rangle_{\mathrm{mol}} \neq|i\rangle_{\mathrm{mol}}$ & $\begin{array}{l}\text { rate of molecular } \\
\text { process, e.g., RET }\end{array}$ & $\begin{array}{c}\text { rate of spectroscopic } \\
\text { process, e.g., MPA }\end{array}$ \\
\hline
\end{tabular}

${ }^{a}$ Only one possibility leads directly to an energy, i.e., when neither radiation nor matter states undergo change. All other possibilities, which relate to offdiagonal matrix elements, serve as probability amplitudes. SHG denotes second harmonic generation; RET is resonance energy transfer; MPA is multiphoton absorption; $|i\rangle$ and $|f\rangle$ are initial and final states, respectively.

excited state decay without incorporating real exponential decay, breaking time-reversal symmetry. Attempts to circumvent the problem generally require an atomic gas assumption, where damping is due only to radiative processes [62-64].

\section{Rates and Energies}

It is important to note an important distinction between rates and energies, in which the QED treatment clarifies an issue that is sometimes obscure in other representations. Clearly, it is possible for the initial and final states in any calculation to be identical, or to differ. When we account for both the Hilbert states of matter and Fock states of the radiation, four possibilities arise (as shown in Table 1), signifying evaluations that are either diagonal, or off-diagonal, in each quantum basis. Failure to explicitly consider the quantized nature of radiation can produce confusion, in particular, over correct usage and distinctions between the matrix elements involved in optically parametric processes and energy evaluations.

For cases where the initial and final states are identical, which can be understood as phenomena rather than processes, only diagonal elements of the matrix element, $M_{i i}$, are significant; the result delivered by computation of Eq. (8) thus represents an expectation value with respect to the operator $T$ [65]. Here, there is no transfer of energy (or linear or angular momentum) between the radiation field and matter. This signifies that the occupancy of each radiation mode is unchanged, as also is the population of each molecular state. In this situation, the calculations are essentially those of time-independent perturbation theory, since both the molecular and radiation states are identical in the initial and final states, and a physically meaningful potential energy $\Delta E$, is identified with the real part of $M_{i i}$. It should be emphasized, however, that the evaluation of such diagonal matrix elements does not preclude their indirect involvement in processes. In nonuniform fields, where the energy of interaction with material particles varies with position-as, for example, in optical trapping - then mechanical motions may arise in response to the associated potential energy surface.

In contrast, when the initial and final states differ, offdiagonal elements arise. Consequently, the physical observable is no longer represented by an energy shift, but by a timedependent rate. Securing the analytical form of the matrix element, $M_{f i}$, enables all of the related observable quantities to be calculated straightforwardly. This is commonly achieved directly from Eq. (8) via Fermi's "Golden Rule" for determining a process rate, $\Gamma$, for an overall system transition $|f\rangle \leftarrow|i\rangle$ [66],

$$
\Gamma=\frac{2 \pi}{\hbar}\left|\sum_{\xi} M_{f i}(\xi)\right|^{2} \rho_{f},
$$

where $\rho_{f}$ signifies the number density of states per unit energy interval. In general, for both QED and SCT, this emerges as a convolution of state densities over the linewidths of the initial and final states of the matter, and of the radiation, commonly reducing in effect to the highest of those four individual densities of state [67]. The appearance of $\rho_{f}$ in Eq. (11) is a consequence of energy conservation between initial and final states, with the latter forming a continuum of levels, and a discrete sum over final states converted to an integral formula. Alternatively, the rate may be written in terms of a delta function, which ensures conservation of energy by the system as a whole [1]. For optical processes such as absorption that involve uncorrelated processes in individual molecules $\xi$, the summation in Eq. (11) can be extracted from the modulus square, and the result reduces to a simple multiplication by the number of molecules in the system. Cross-terms arise where coherence holds sway, as we shall see in the example of second-harmonic generation (SHG) in the next section.

Consistent with the time-dependent perturbation approach, the above form of result is regarded as applicable at times beyond the scale of the rapid quantum oscillations that occur at optical frequencies. (Alternatively, in cases where Rabi oscillations occur-usually limited to systems comprising simple particles with discrete energy levels - the matrix element feeds into a time-dependent rate cast in a different form; see, for example, Ref. [68]). The range of optical processes for which Eq. (11) can be applied includes those where both material and radiation change state, as well as those in which the radiation alone changes (parametric interactions such as optical harmonic generation and frequency mixing) and also those where it is only matter that changes (as, for example, in resonance energy transfer $(\mathrm{RET}))$. It is worth observing that, even in the presence of saturation, or other changes in state populations affecting the measured rate of a collective, the above rule holds for each individual molecule.

It is interesting to observe another substantial difference in operation between the SCT and QED theories, readily illustrated by reference to simple absorption. In the realm of SCT, reference is frequently made to a "rotating-wave approximation," RWA. This premise is used to discard certain terms in the derivation of Fermi rule rates, on the basis of ultrafast oscillation averaging to zero over experimentally meaningful times. Consider an excitation transition $m \leftarrow 0$, for example, where the excitation energy is within a linewidth of the input photon energy, i.e., $E_{m 0} \approx \hbar \omega$, and $\omega=c k$ is the circular frequency of the optical input. Theory based on a classical treatment of the radiation - see, for example, the standard treatment given in Ref. [69]—produces two quantum amplitude terms, one featuring the phase factor $\exp \left[i\left\{\left(E_{m 0} / \hbar\right)-\omega\right\} t\right]$ at time $t$, and the other, a phase factor $\exp \left[i\left\{\left(E_{m 0} / \hbar\right)+\omega\right\} t\right]$. The latter term is generally discarded on RWA arguments, since it oscillates around zero with a frequency around $2 \omega$. However, in 
the QED representation, the Heisenberg equation of motion for operators reveals that the photon annihilation and raising operators, $a^{(\eta)}(\boldsymbol{k})$ and $a^{\dagger(\eta)}(\boldsymbol{k})$, carry temporal phase factors $\exp [-i \omega t]$ and $\exp [+i \omega t]$, respectively. It then becomes evident that the latter can never contribute to absorption because it corresponds to an upward transition accompanied by the emission, not absorption, of a photon. The RWA need never be invoked; any supposedly additional terms would contravene energy conservation and are, therefore, never experimentally measurable. In passing, we note that the Heisenberg picture can offer calculational advantages and insights [70], afforded by operator equations of motion resembling those of classical mechanics $[8,71]$, but in the course of most applications it is the interaction representation, presented in this review, that proves most expedient. In this representation, state amplitudes are cast in a form that is time-independent when interaction terms are absent [72].

\section{Time-Ordered Diagrams}

To facilitate computation of the matrix element, especially in QED treatments of nonlinear optical processes [5,7] and also for intermolecular interactions [5,8,73], it is convenient to draw time-ordered diagrams to depict the coupling of electromagnetic radiation with matter via individual photon creation and annihilation events, and any concomitant changes in the state of the molecules. Conventionally, these depictions are constructed as analogs of the Feynman diagrams employed to great effect in evaluating pathway sums in quantum field theory [74]. All possible time-ordered sequences that link prescribed initial and final states must again be entertained in nonrelativistic QED theory. Any individual diagram corresponds to a contributory term of the quantum amplitude in the determination of a given measurable. A more recent diagrammatic development, known as a state-sequence diagram [75], combines the complete set of interaction sequences into a single representation; this approach will be illustrated in Section 4.

In addition to aiding computation, the complete set of diagrams for a given overall interaction provides a visual representation of the physical process under consideration, enhancing understanding: example diagrams will be given when applications are examined below. The depiction of specific photon absorption and emission events and sequences, which allows the evolution of the total system to be easily followed, contrasts with the conventional figurative representation of radiation-matter interactions in terms of primitive energy-level diagrams. An obvious major drawback of the latter schemes is that it generally conceals the significance of many terms arising from application of the perturbation theory formalism. More subtly, the depiction on an energy-scale diagram, of any process beyond simple photon absorption or emission, usually implies a specific sequence of interactions; see, for example, a comparison for the case of Rayleigh scattering shown in Fig. 1. In QED, the mathematics specifically requires considerations of all possible time sequences, making proper allowance for the consequences of quantum uncertainty. Moreover, in any calculation that entails two or more different pathways, interferences in the associated matrix elements duly represent quantum coherence effects [43].

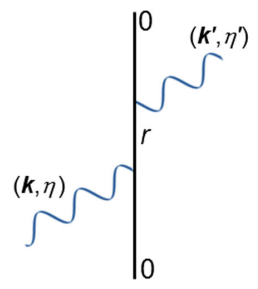

(a)

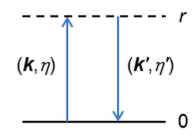

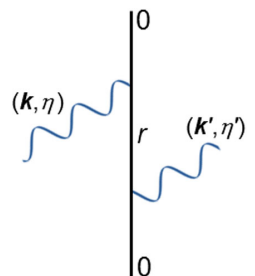

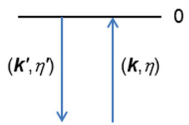

(b)
Fig. 1. Nonforward Rayleigh scattering. Top, two time-ordered diagrams; bottom, their energy level equivalents. In the time-ordered diagrams, the vertical line represents the molecule and wavy lines the input and output photons; 0 and $r$ denote ground and virtual intermediate states, respectively, time progressing upwards. (a) Input photon is annihilated, then output photon is created; (b) output photon is created, then input photon is annihilated. To correctly derive the matrix element, and hence the correct dependence on polarizability, both sequences must be included in the calculations, whether in QED or SCT. Underneath the Feynman diagrams are the corresponding energy level figures, (a) as usually depicted, and (b) its seldom-shown counterpart that lacks conventional interpretation, wrongly indicating negative energies.

\section{E. Order and Phase}

Across the electromagnetic spectrum, the character of material response to phase is strongly influenced by the degree of order, most particularly within the span of a wavelength, within or at the surface of the material. In the electromagnetic region of optical wavelengths $100 \mathrm{~nm}$ to $1 \mathrm{~mm}$, the most prominent manifestations of phase lie in interference phenomena, and in corresponding differences between coherent and incoherent phenomena.

For optical processes, rate equations derived from the modulus square of the quantum matrix element primarily apply to radiation that is propagating in a direction fixed relative to the positions and orientation of one or more material particles. This holds for processes involving absorption and/or stimulated emission of one or more photons, whether from one or many beams. Even for spontaneous emission from a single fixedlocation source, producing radiation that is spatially distributed according to the dipolar nature of the emission, there may be a specific orientational disposition with respect to a direction of observation. However, to obtain results for more extended but isotropic systems, such as most liquids, solutions or gases-or condensed phase samples that have truly random local orderusually requires that a free orientational average be carried out [76]. Here, since the property of isotropy relates to the bulk, it is appropriate to exploit the ergodic theorem, which dictates that the time-averaged response of any single center, under equilibrium conditions, equates to the ensemble average. This aspect of theory arises equally under SCT or QED formulations. 
The primary (E1) contributions to the rate equation for an incoherent (non-wave-vector conserving) process involving $q$ photons entail tensor orientational averages of rank $2 q$, or $q$, if the process is coherent [7]; any significant involvement of higher-order multipoles will often generate additional terms of higher (including odd) ranks. General results are known for averages up to the eighth rank [77], while results for special cases are known up to the eleventh rank [78-80]. Explicit averaging procedures are most readily performed by expressing the directional properties of external radiation, and the response it induces in the material system, in terms of Cartesian tensors whose components are referred to space- and body-fixed frames of reference, respectively. Accordingly, the necessary orientational averages can be performed in terms of a rotational average of the two corresponding Cartesian frames. Working with Cartesian coordinates affords particular advantages for optical interactions: the material response may thereby have reference to intrinsic axes and planes of symmetry, readily connecting with spectroscopic selection rules; equally, radiation parameters such as wave vectors and polarization vectors can be referred to experimentally designed configurations on an optical table.

As we saw in Eq. (11), the matrix element typically comprises a sum of terms for each of the $N$ component molecules or electronically distinct optical centers. Each contribution carries a local phase, arising from the product of phases in each of the distinct photon interactions. For example, in a frequencydoubling process involving a set of molecules (or other centers), each labeled $\xi$, positioned at $\boldsymbol{R}_{\xi}$, this product takes the form $\mathrm{e}^{i\left(2 \boldsymbol{k}-\boldsymbol{k}^{\prime}\right) \cdot \boldsymbol{R}_{\xi}} \equiv \mathrm{e}^{i \boldsymbol{k} \cdot \boldsymbol{R}_{\xi}} \times \mathrm{e}^{i \boldsymbol{k} \cdot \boldsymbol{R}_{\xi}} \times \mathrm{e}^{-i \boldsymbol{k}^{\prime} \cdot \boldsymbol{R}_{\xi}}$, where $\boldsymbol{k}$ is the wave vector for each input photon, $\boldsymbol{k}^{\prime}$ for the output. The individual phases in this product simply arise from the corresponding annihilation and creation terms in the field expansion [Eq. (7)]. For $N$ active centers, the rate expression therefore contains, in principle, $N^{2}$ terms: there are $N$ "diagonal" terms collectively representing the response of individual molecules, and $N(N-1)$ "off-diagonal" terms arising from the interference of matrix elements for molecules at different locations. The topic of correctly dealing with randomly positioned scatterers is neatly introduced in a classic text by Marcuse [81].

Since each diagonal term arises from the modulus square of an individual amplitude, phase information disappears and, on average, each of the $N$ results is the same. These terms hence signify an additive, incoherent response, contributing a rate that scales linearly with the material's optical density. The off-diagonal interference terms average to zero, except in one very special case: when the phase of each matrix element is itself zero. For example, in the frequency-doubling illustration given above, fulfilling the SHG wave-vector matching condition $2 \boldsymbol{k}-\boldsymbol{k}^{\prime}=0$ ensures that there can be coherent addition of all the interference terms and, in consequence, the rate essentially has a quadratic dependence on optical density. Equally, for the theory, this signifies that the matrix elements for the process can be rotationally averaged prior to their addition and squaring. Physically, such phase-matching corresponds to conservation of photon linear momentum by the radiation field alone. However, under any other condition (such that there is some momentum transfer to each material component, e.g., where the harmonic is not collected in the forward direction but at some other angle), the coherent terms net to zero. The process
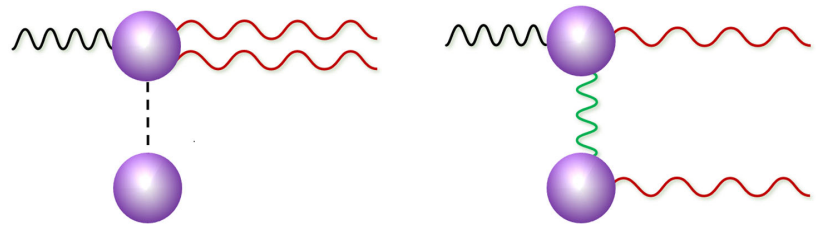

Fig. 2. Degenerate downconversion, two of several mechanisms involving more than one optical center in the conversion process. Optical input is from the left, output to the right, and wavy lines denote photons. Left-hand case, the ancillary unit is essentially coupled into the process through a static interaction (dashed line), which means that no energy is transferred between the centers. Right-hand case, dynamic coupling, which does involve energy migration, is mediated by a virtual photon (green wavy line). Adapted from Ref. [88].

is accordingly governed by the fewer remaining, incoherent rate terms, in this case associated with the much weaker process known as hyper-Rayleigh scattering [82-87].

Other, more intricate possibilities also arise. Where multicenter interactions occur, a simple reduction of the rate according to Eq. (11) may need correction. For coherent interactions, it can become necessary to sum a matrix element over a large ensemble of mutually coupled material centers, in the sense that each center experiences a local electronic environment that is influenced by electrodynamic and electrostatic fields due to its neighbors. Formally, any such form of interaction engages the components in virtual transitions. An example that has received recent interest is degenerate frequency downconversion, $2 \omega \rightarrow \omega+\omega$, primarily mediated by a three-interaction matrix element $M_{f i}^{(3)}$ that corresponds to $T^{(3)}$ in Eq. (8).

Understanding the overall nature of the conversion process, it is readily observed from Fig. 2 that any ancillary optical centers in the bulk may participate in such optically parametric processes in a variety of ways (a more complete set is identified in a recent publication [88]). Significantly, when such centers undergo no lasting change in electronic state, there is no limit on how many of them can participate, subject, of course, to the sharply dropping effectiveness of the retarded (virtual photon) coupling with increasing distance, which means that nearneighbors play the most prominent role. In the development of the quantum amplitude from higher-order terms of the transition operator [Eq. (8)], care therefore has to be taken with the implicitly introduced use of the completeness relation identity, which for an $N$-element system must now be cast as

$$
1=(N-1)^{-1} \sum_{q \neq \mathrm{A}}^{N-1} \sum_{r}\left|r\left(\xi_{q}\right)\right\rangle\left\langle r\left(\xi_{q}\right)\right| .
$$

Here, A signifies any of the optical centers where input photons are annihilated, and $r$ is a generic label for an intermediate state.

Finally, for processes that necessarily engage concerted photon interactions with coupled molecules, each matrix element may engage an irremovable phase dependence on the mutual separation vector, necessitating a phased orientational average [89]. Another frequently encountered case, also requiring a special type of averaging process, occurs when dealing with anisotropic media. This is dealt with by a method closely related to the phase averaging just described, but which has a real 
instead of an imaginary argument in a weighting exponential factor. An example is provided by the action of a static electric field, $\boldsymbol{E}_{0}$, on a polar fluid characterized by permanent electric dipoles, $\boldsymbol{\mu}_{0}$, leading to the inclusion of a temperature dependent Boltzmann-weighting factor of the form $\mathrm{e}^{-\boldsymbol{\mu}_{0} \cdot \boldsymbol{E}_{0} / k_{B} T}$ in the orientational average of the ensemble, where $T$ is the absolute temperature and $k_{B}$ is Boltzmann's constant.

\section{COMPARISON OF THE TWO THEORIES IN APPLICATION}

Before examining and comparing specific light-matter interactions in both the SCT and QED points of view, it is worth observing that, due to SCT commonly being the default theory, many optical phenomena are often cast in such terms. A clear advantage of QED methods at a more fundamental level is that it can be cast in terms of the PZW Hamiltonian, that is, a gaugeindependent quantum Hamiltonian that couples the multipole transition moments of the matter (E1 and M1, E2, and so on) directly to the physical electromagnetic fields, and automatically accounts for all intermolecular Coulombic interactions [9]. In contrast, SCT often invokes the minimal-coupling Hamiltonian, where light-matter interactions are described in the much more physically unintuitive vector potential and canonical momentum variables, include a separate potential energy for Coulombic interactions, and for any multiphoton process require computation of an additional term that is quadratic in the vector potential. Although it is possible to construct a semiclassical interaction Hamiltonian of E1 form, Woolley (and others [90]) has highlighted a widely underappreciated fact that even the notion of the ubiquitous dipole coupling is in fact unsupported by classical wave theory, and multipolar descriptions of light-matter interactions of any order are only rigorously defensible within a fully quantized framework [91,92]. In this context, it is perhaps worth recalling that the multipolar Hamiltonian of QED can itself be secured by applying a unitary transformation (the PZW transformation) to the minimal-coupling Hamiltonian $[9,27,28]$.

As examples to compare SCT and QED, we now examine two types of mechanisms: process rates, exemplified by SHG, and optically induced energies, focusing on optical binding.

\section{A. SHG}

To appreciate the origin of optical properties in any medium entails the development of theory to elicit the detailed electronic behavior of the material under the influence of electromagnetic radiation. Standard introductions to optical nonlinearity extend a simple SCT formulation of linear response to an applied electric field - a response whose nature is considered to be the induction of a polarization field (comprising individual dipole moments) —on the grounds that this presents only an approximation to the true response of many materials. When such a field is oscillatory, as in the optical case, it is reasoned that the correction terms associated with nonlinear response are responsible for the generation of optical harmonics, i.e.,

$$
P_{i}=\varepsilon_{0}\left\{\chi_{i j}^{(1)} E_{j}+\chi_{i j k}^{(2)} E_{j} E_{k}+\chi_{i j k l}^{(3)} E_{j} E_{k} E_{l}+\ldots\right\},
$$

which represents component $i$ of a vector "electric polarization" $\boldsymbol{P}$ for the material, $\boldsymbol{E}$ is the applied electric field, and $\chi^{(q)}$ is the $q$ th-order electric susceptibility with the status of a $(q+1)$ rank tensor, as denoted by the number of indices. The above expression again adopts the convention of implied summation, on a Cartesian set, over repeated indices. From the semiclassical perspective, the electromagnetic radiation is considered to be a wave varying sinusoidally with frequency $\omega$ over time $t$, namely,

$$
E(t)=E_{0} \cos \omega t,
$$

where $E_{0}$ is the electric field amplitude of the light wave. Inserting this expression into Eq. (13) gives the result:

$$
\begin{aligned}
P_{i}(t)= & \varepsilon_{0} \chi_{i j}^{(1)} E_{0 j} \cos \omega t+\frac{\varepsilon_{0}}{2} \chi_{i j k}^{(2)} E_{0 j} E_{0 k}(1+\cos 2 \omega t) \\
& +\frac{\varepsilon_{0}}{4} \chi_{i j k l}^{(3)} E_{0 j} E_{0 k} E_{0 l}(3 \cos \omega t+\cos 3 \omega t)+\ldots
\end{aligned}
$$

The first correction term to the linear response, i.e., the second term of Eq. (15), is thus considered the source of an optical second harmonic $2 \omega$; the next correction is deemed the origin of a third harmonic, and so on. To derive the detailed structure of the linear and nonlinear susceptibilities, one usually has recourse to essentially prescriptive methods in a form first proposed by Ward [93]. A similar cast of expression applies at the level of individual molecules, whose net effect (subject to local field effects and cascade processes) gives rise to the bulk linear and nonlinear susceptibilities.

There are many reasons, some delineated in more detail elsewhere [7], why the above description is unsatisfactory. For example, Eq. (15) logically implies that a medium with a nonvanishing $\chi^{(2)}$ will always generate some amount of second harmonic, even at intensity levels that would correspond to only one photon of input, whereas, of course, energy conservation requires two input photons for every second-harmonic photon that is produced. This fault in SCT directly owes its origin to the representation of the optical field by a classical wave, a description that makes no provision for quantum limits. It is, in fact, simple and instructive to show that the statistical probability of two photons arriving together leads to the observed quadratic dependence on intensity [94]. Other, commonly overlooked problems with the semiclassical approach are its implicit (but in SCT, mathematically unjustified) rejection of terms such as the unity in the second "SHG" term, which in fact signifies an entirely different process: here, the linear electro-optic effect [95], entirely unrelated to SHG. Moreover, the fact that the modulus square of the polarization is deemed to represent an observable signal is complicated by the fact that the resulting cross-terms have no physical significance: they would signify energy nonconserving interference. It is noteworthy that the semiclassical rationale for the emergence of a second harmonic, Eq. (15), is based on the assumption of quadratic response, and use of the simple trigonometric identity $\cos ^{2} \omega t=\frac{1}{2}(1+\cos 2 \omega t)$. A similar logic applies for sum-frequency generation (SFG).

A photon-based foundation, based on QED, for describing nonlinear optical phenomena presents none of these difficulties. For example, no cross-terms of the kind discussed above arise, 
precisely because different processes are associated with different radiation state transitions in the radiation field. Quantum theory requires that quantum amplitudes are added only for processes that connect the same pair of initial and final states of the system. This means that only one leading component of the transition operator [Eq. (8)] is required for a given process, according to the number of light-matter interactions involved; for instance, only $T^{(1)}$ is required for one-photon emission, $T^{(2)}$ for two-photon absorption and $T^{(3)}$ for SHG (two photons in and one out).

Reinforcing this point, we now examine the QED derivation of the SHG signal intensity [96,97]. As is commonly known, SHG involves the concurrent absorption of two photons of frequency $\omega$ at a molecule, along with emission of a single photon at double the frequency, so that $2 \omega=\omega^{\prime}$; the molecule remains in the same quantum state, while the radiation changes state. This, as indicated earlier, enables the wave-vector matching condition $2 \boldsymbol{k}-\boldsymbol{k}^{\prime}=0$. The starting point, in general, amounts to no more than clearly identifying the initial and final states for each instance of the fundamental interaction. This means defining the optical radiation mode and representative material components that are involved, and identifying their initial and final states. For SHG, the initial $|i\rangle$ and final $|f\rangle$ states are $\left|E_{0}^{\mathrm{A}} ; n(\boldsymbol{k}, \eta), 0\left(\boldsymbol{k}^{\prime}, \eta^{\prime}\right)\right\rangle$ and $\left|E_{0}^{\mathrm{A}} ;(n-2)(\boldsymbol{k}, \eta), 1\left(\boldsymbol{k}^{\prime}, \eta^{\prime}\right)\right\rangle$, respectively, which denotes molecule $A$ remaining in its ground state (subscript 0 ) and two photons of one radiation mode converting into one photon of the other state. By linking the initial to the final state, a full set of topologically distinct timeordered Feynman diagrams can be constructed or, even more expediently (especially for more complicated mechanisms, such as the one discussed next), a single state-sequence diagram that assimilates the information content of the entire Feynman set [75].

By analyzing the time-ordered diagrams of SHG (Fig. 3), or its equivalent single state-sequence diagram (Fig. 4), the three routes from the initial to the final state can be visualized, and the virtual intermediate states can be fully identified (Table 2). These three channels within the state-sequence diagram are represented in Table 3; the relative efficiency of each of these channels, for a given input frequency, is developed elsewhere [98]. Since SHG involves three light-matter interactions, as shown by these diagrams, the $T^{(3)}$ term of Eq. (8) is solely employed (which contrasts with SCT). Therefore, since the completeness relation $|r\rangle\langle r|=\mathbf{1}| s\rangle,\langle s|=\mathbf{1}$ are used, and $H_{0}$ operates on virtual intermediate states $|r\rangle$ and $|s\rangle$ to produce eigenvalues $E_{r}$ and $E_{s}$, the following expression is generated from Eq. (8):

$$
M_{f i}=\sum_{r, s} \frac{\left\langle f\left|H_{\text {int }}\right| s\right\rangle\left\langle s\left|H_{\text {int }}\right| r\right\rangle\left\langle r\left|H_{\text {int }}\right| i\right\rangle}{\left(E_{i}-E_{r}\right)\left(E_{i}-E_{s}\right)}
$$

where the summation is over the intermediate states identified in Table 2. However, it is noteworthy that three terms (not four) will arise from Eq. (16), which is apparent because Fig. 4 has two boxes that are not linked, which means that a fourth channel (and a fourth term) is not possible.

The next step is to insert the explicit form of each quantum state and its corresponding energy, provided by Table 2 (with the knowledge that one route, thus one term, does not exist)—along

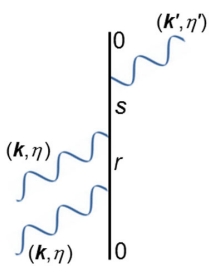

(a)

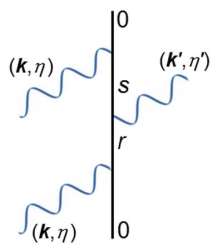

(b)

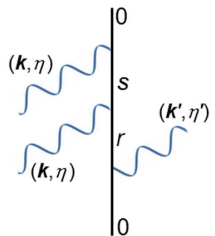

(c)
Fig. 3. Time-ordered diagrams for SHG, where $r$ and $s$ denote the virtual intermediate states. (a) Two input photons are annihilated, then an output photon is created; (b) an input photon is annihilated, then an output photon is created, then an input photon is annihilated; (c) an output photon is created, then two input photons are annihilated.

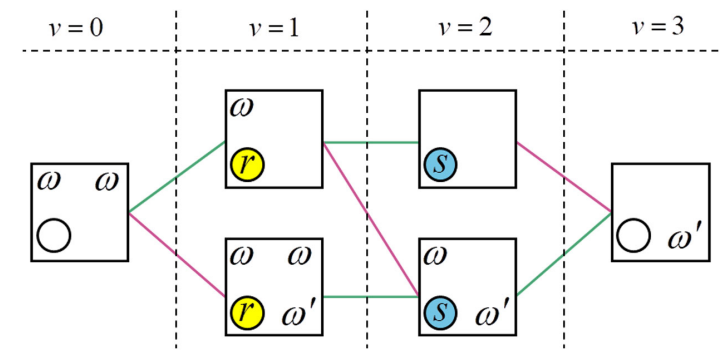

Fig. 4. State-sequence diagram for SHG, in which the initial and final states are on the left- and right-hand sides of the diagram, respectively; the four possible intermediate system states are in the center, and the virtual intermediate molecular states are filled circles. Green and purple lines denote photon annihilation and creation, respectively, going from left to right. Note that $\omega=c k$ and $\omega^{\prime} \equiv c k^{\prime} ; v$ is the step number.

Table 2. System States Decomposed into Molecule and Radiation States, and Their Corresponding Energies for $\mathrm{SHG}^{a}$

\begin{tabular}{lcc}
\hline System State $\left|\boldsymbol{r}_{v}^{l}\right\rangle$ & $|\mathbf{m o l}\rangle|\mathbf{r a d}\rangle$ & Energy \\
\hline$\left|r_{0}^{1}\right\rangle \equiv|i\rangle$ & $\left|E_{0} ; n, 0\right\rangle$ & $E_{0}+n \hbar \omega$ \\
$\left|r_{1}^{1}\right\rangle \equiv|r\rangle$ & $\left|E_{r} ; n-1,0\right\rangle$ & $E_{r}+(n-1) \hbar \omega$ \\
$\left|r_{1}^{2}\right\rangle \equiv|r\rangle$ & $\left|E_{r} ; n, 1\right\rangle$ & $E_{r}+n \hbar \omega+\hbar \omega^{\prime}$ \\
$\left|r_{2}^{1}\right\rangle \equiv|s\rangle$ & $\left|E_{s} ; n-2,0\right\rangle$ & $E_{s}$ \\
$\left|r_{2}^{2}\right\rangle \equiv|s\rangle$ & $\left|E_{s} ; n-1,1\right\rangle$ & $E_{s}+(n-1) \hbar \omega+\hbar \omega^{\prime}$ \\
$\left|r_{3}^{1}\right\rangle \equiv|f\rangle$ & $\left|E_{0} ; n-2,1\right\rangle$ & $E_{0}+(n-2) \hbar \omega+\hbar \omega^{\prime}$ \\
\hline
\end{tabular}

${ }^{a}$ Here, $v$ is the step number and $l$ the vertex number (defined from Fig. 4). For SHG, the initial and final energies are identical, since $\hbar \omega^{\prime}=2 \hbar \omega$.

with $H_{\text {int }}$ given by Eq. (6) - into Eq. (16). Here, $\boldsymbol{\mu}$ operates on the molecular states and $\boldsymbol{d}^{\perp}$ onto the radiation states; remember that only the first or second term of Eq. (7) is necessary, depending on whether $H_{\text {int }}$ relates to photon annihilation or creation, respectively. Therefore, for a molecule $\xi$ subject to a laser beam containing $n$ photons, we find that

$M_{f i}=-\frac{i}{2}\left(\frac{\hbar \omega}{\varepsilon_{0} V}\right)^{3 / 2} n \bar{e}_{i}^{\left(\eta^{\prime}\right)}\left(\boldsymbol{k}^{\prime}\right) e_{j}^{(\eta)}(\boldsymbol{k}) e_{k}^{(\eta)}(\boldsymbol{k}) \sum_{\xi} \beta_{i j k}(\xi)$,

in which $n \approx n-1$ is assumed for an input laser with large $n$. The assumption of wave-vector matching removes any 
Table 3. Three Channels for Traveling from the Initial State $\left|r_{0}^{1}\right\rangle$ to the Final State $\left|r_{3}^{1}\right\rangle$ in the State-Sequence Diagram of Fig. 4

\begin{tabular}{lccc}
\hline Channel & Sequence & Mode Occupancy & Figure \\
\hline 1 & $\left|r_{0}^{1}\right\rangle \rightarrow\left|r_{1}^{1}\right\rangle \rightarrow$ & $(\omega, \omega) \rightarrow(\omega) \rightarrow$ & $3(\mathrm{a})$ \\
& $\left|r_{2}^{1}\right\rangle \rightarrow\left|r_{3}^{1}\right\rangle$ & ()$\rightarrow\left(\omega^{\prime}\right)$ & \\
2 & $\left|r_{0}^{1}\right\rangle \rightarrow\left|r_{1}^{1}\right\rangle \rightarrow$ & $(\omega, \omega) \rightarrow(\omega) \rightarrow$ & $3(\mathrm{~b})$ \\
& $\left|r_{2}^{2}\right\rangle \rightarrow\left|r_{3}^{1}\right\rangle$ & $\left(\omega, \omega^{\prime}\right) \rightarrow\left(\omega^{\prime}\right)$ & \\
3 & $\left|r_{0}^{1}\right\rangle \rightarrow\left|r_{1}^{2}\right\rangle \rightarrow$ & $(\omega, \omega) \rightarrow$ & $3(\mathrm{c})$ \\
& $\left|r_{2}^{2}\right\rangle \rightarrow\left|r_{3}^{1}\right\rangle$ & $\left(\omega, \omega, \omega^{\prime}\right) \rightarrow$ & \\
& & $\left(\omega, \omega^{\prime}\right) \rightarrow\left(\omega^{\prime}\right)$ & \\
\hline
\end{tabular}

molecule-specific phase factor, as we discussed in Section 3.E. The explicit form of the hyperpolarizability tensor, $\beta_{i j k}$, is given by

$$
\begin{aligned}
\beta_{i j k}= & \sum_{r, s}\left(\frac{\mu_{i}^{0 s} \mu_{j}^{s r} \mu_{k}^{r 0}}{\left(E_{s 0}-2 \hbar \omega\right)\left(E_{r 0}-\hbar \omega\right)}\right. \\
& +\frac{\mu_{j}^{0 s} \mu_{i}^{s r} \mu_{k}^{r 0}}{\left(E_{s 0}+\hbar \omega\right)\left(E_{r 0}-\hbar \omega\right)} \\
& \left.+\frac{\mu_{j}^{0 s} \mu_{k}^{s r} \mu_{i}^{r 0}}{\left(E_{s 0}+2 \hbar \omega\right)\left(E_{r 0}+\hbar \omega\right)}\right) .
\end{aligned}
$$

A simpler QED method to determine nonlinear optical response tensors of this type, which is equally applicable for much more complicated processes, can be found elsewhere [99]. In passing, we note the implications of including damping factors in the energy denominators, notwithstanding the difficulties identified in Section 3.B for both QED and SCT applications. It is gratifying that the issue of the sign on the damping correction terms has proven to have no discernible effect on the dispersion behavior of SHG conversion in the wings of either a single- or a two-photon resonance [100,101].

The second-harmonic emission rate may be obtained from the Fermi Golden Rule (11), and an orientational average performed to yield the rate for a fluid sample. On the assumption that there is no orientational correlation between different optical centers, the second-harmonic radiant intensity $I$ (for a sample containing $N$ molecular scatters) may be obtained from this rate and written as

$$
I=\frac{\bar{I}_{0}^{2} g^{(2)} k^{4} N^{2}}{72 \pi^{2} \varepsilon_{0}^{3} c}\left|\varepsilon_{i j k} \bar{e}_{i}^{\left(\eta^{\prime}\right)}\left(\boldsymbol{k}^{\prime}\right) e_{j}^{(\eta)}(\boldsymbol{k}) e_{k}^{(\eta)}(\boldsymbol{k}) \varepsilon_{\lambda \mu \nu} \beta_{\lambda \mu \nu}\right|^{2},
$$

where $\bar{I}_{0}$ is the mean irradiance of the input beam, $g^{(2)}=$ $\langle n(n-1)\rangle /\langle n\rangle^{2}$ is the degree of second-order coherence, reflecting the absorption of two photons of the same mode, which need not necessarily be represented by a number state, $\varepsilon_{i j k}$ is the Levi-Civita alternating tensor, and Greek subscripts refer to Cartesian tensor components in the molecule-fixed frame. Equation (19) leads to the well-known result that the second-harmonic signal intensity vanishes for a randomly oriented source. It is a special case of the general result that for all multipole moments, the intensity is zero for coherent generation of even harmonics. A clear advantage is afforded by the microscopic treatment of the phenomenon by way of QED theory, relative to descriptions in terms of the nonlinear polarization that only identify the coherent contribution [102].

\section{B. Optical Binding}

Outside of nonlinear optical processes, the techniques of QED are equally adept when applied to other forms of phenomena that involve light-matter interactions. An interesting example is the energy shift (the origin of an optical force) that arises when an off-resonant beam of sufficient intensity irradiates a pairwise molecular system [103,104]; this is known as optical binding. Strictly, as detailed earlier, this is not a process: the Fermi rate is replaced by an expression, which determines the energy shift, that takes the real part of the matrix element only [65]. This optical binding mechanism is much more complicated than the previous case of SHG because it involves two molecules interacting with input, output, and mediating photons. In cases such as these, it makes more sense to apply the state-sequence diagram only (Fig. 5) since 24 channels are involved; thus, 24 time-ordered diagrams are required to depict the same mechanism. In fact, another state-sequence diagram (or another 24 time-ordered diagrams) is required to completely describe optical binding, i.e., for the cases where the two molecules are interchanged. To derive the expression for the energy shift $\Delta E$, the $T^{(4)}$ term of Eq. (8) is used so that we eventually determine

$$
\Delta E=\left(\frac{n \hbar c k}{\varepsilon_{0} V}\right) \operatorname{Re}\left\{e_{i}^{(\eta)}(\boldsymbol{k}) \bar{e}_{l}^{\left(\eta^{\prime}\right)}\left(\boldsymbol{k}^{\prime}\right) \alpha_{i j}^{\mathrm{A}} V_{j k}(k, \boldsymbol{R}) \alpha_{k l}^{\mathrm{B}} \mathrm{e}^{i \boldsymbol{k} \cdot \boldsymbol{R}}\right\},
$$

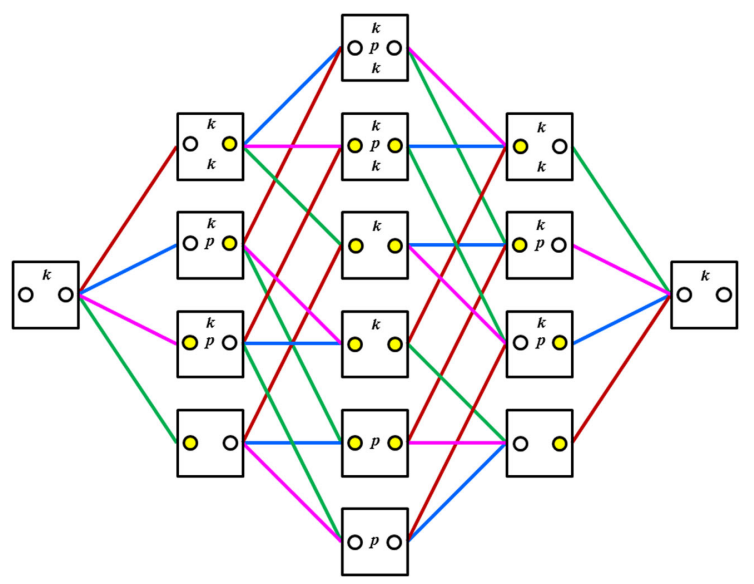

Fig. 5. State-sequence diagram for optical binding, in the center of which 14 possible intermediate states are present. In each box, the leftand right-hand circles are molecules $\mathrm{A}$ and $\mathrm{B}$, respectively; an empty circle is an unexcited molecule, and a filled circle denotes a virtual intermediate state; $\boldsymbol{k}$ is a photon relating to the irradiating beam; $\boldsymbol{p}$ is a mediating photon that couples A and B. Represented by a line between the boxes, one of four events are possible: (i) laser photon annihilated at A (green line); (ii) mediating photon created at A (orange line); (iii) mediating photon annihilated at B (blue line); or (iv) laser photon created at B (purple line). The order in which these events arise is the whole basis for the diagram and the 24 channels from initial to final state (from left to right). Each channel has a unique time-ordered diagram equivalent. 
where $\alpha_{i j}$ is a polarizability tensor, $V_{j k}(k, \boldsymbol{R})$ is the dipoledipole coupling tensor (in the E1 approximation) that represents virtual photon exchange between molecules A and $\mathrm{B}$, and $\mathrm{e}^{\boldsymbol{i} \boldsymbol{k} \cdot \boldsymbol{R}}$ is a phase factor that arises because the emission and absorption events occur at separate locations, with relative displacement vector $\boldsymbol{R}$.

The same energy shift result could be obtainable by using SCT, but the method is much more physically ambiguous and less rigorous. It works on the principle that the oscillatory electromagnetic field of the input radiation produces motions in the charge distributions of the molecules it encounters. Such motions lead to corresponding oscillating electric dipoles, whose phase is determined by that of the input radiation at each molecule. For two molecules in close proximity, the interactions between their oscillating dipoles are dependent on the relative phase of the optical field at the two locations; it is also subject to the sharp decline of such an interaction with separation. From such a physical picture, one can surmise the physical existence of an optically induced force that exhibits a strongly damped oscillatory dependence on the separation of the molecular pair. Moreover, for particles larger than the wavelength of the input light, it is the classical Lorenz-Mie formalism that should be applied [105-109]. Such microparticles are usually modeled in the ray optics regime and typically involve Mie scattering, in which the input light undergoes internal refraction, leading to an optical force that acts towards the beam center [110].

Beyond the contact region, where individual molecular orbitals significantly overlap, it emerges that these intermolecular binding forces may considerably "override" the intrinsic Casimir-Polder (dispersion) force between molecules. (Notably, the latter itself was derived directly from QED theory by Casimir and Polder, who showed the emergence of an inverse-seventh power distance dependence in the far-zoneagain associated with the fleeting presence of "virtual" photons between the molecules [111].) Most significant is the capacity for multiple molecules to be held together by the input light in stable and noncontact arrays of varying geometries [112-114]. For these intricate systems, the complexity of the optical binding theory quickly escalates. Nevertheless, a considerable amount of groundwork has been accomplished, leading to the mapping of potential energy landscapes [115-117]. Within such energy surfaces, the molecules reside in the minima, at positions where the optical binding force is null, so that certain stable arrangements can be maintained with fixed separations. This feature enables optical self-assembly, driven by optical binding forces. A clear example of the powerful predictive capacity of QED is that optical binding was originally predicted by Thirunamachandran using QED methods [118], almost a decade before it was first observed experimentally [119]. Further QED studies concerned with optical forces have highlighted discriminatory trapping and binding forces that offer novel possibilities for separating enantiomers by all-optical means $[48-50,120,121]$. A review on the latest research on such binding forces at the nanoscale is to be found in Ref. [122].

Previous sections aimed to illustrate the power and rigor of applying QED methods. However, one of the virtues of using such procedures is the unnecessary rehearsal of calculations on every application, nor to verify afresh their robust validity. In practice, therefore, one can move quite swiftly from a formulaic representation of a process of interest to a full-fledged calculational implementation. In fact, once the derivations of QED expressions are fully understood, the form of expressions, such as Eq. (20), can be quickly predicted. In the present case, for example, molecules A and B are each associated with one input and one output photon (identical to Rayleigh scattering) that corresponds to a polarizability tensor. The coupling tensor always arises when two molecules are coupled by a mediating virtual photon $[8,123,124]$, while the polarizations of the laser field, phase factor, and the premultiplier arise directly from Eq. (7).

\section{PREDICTIVE CAPACITY OF QED IN OPTICS}

In a general comparison of the predictive capacities of QED with SCT, there are several distinct categories: first, there are cases where SCT unequivocally fails, and QED has to be employed, as, for example, with spontaneous emission, and as we have seen in previous sections. Then there is another category, where SCT delivers results for some optical interactions that are extremely similar to QED theory, though perhaps lending each a different physical insight. Furthermore, there is also a surprising range of phenomena that, while they might indeed have been formulated using SCT, were never envisaged by users of that framework; instead, they were conceived and brought to the fore using QED methods. In addition, one might say that there are, occasionally, conjectured forms of optical interaction where theories based on SCT and on QED lead to apparently different predictions, but where experimental studies have yet to clearly vindicate either theory. The unprecedented success of QED across all of its wider spheres of application to dateexperiments have never yet failed to resolve any dispute in favor of QED—strongly suggests the likely nature of the outcome.

One instance of the first category is spontaneous emission, as we have seen. It is not only important as an example that undermines SCT and justifies QED because of its simple and fundamental importance; it also provides the underlying framework for understanding a multitude of other optical phenomena. For light-matter interactions in general, processes that spontaneously generate new frequencies of light generally require quantum theory: all such processes stem from spontaneous emission of new modes of light, which can only be explained by quantum methods. With this in mind, it is therefore easy to understand why SCT gives near-identical results as QED for processes like single-photon absorption [125], where no modes are spontaneously generated in the processes. However, when one takes into account the discrete quantum structure and energy levels of atoms and molecules, it becomes obvious that light-matter interactions described in terms of discrete quanta of light - photons — offer a much clearer physical understanding than an incident classical wave or classical particle; only the photon concept correctly incorporates the wave-particle duality of light. We now focus on the differences in perspective of the two theories, and the original predictions ofQED.

\section{A. Differences in Perspective}

One of the many important areas of theory to arise primarily from SCT studies is optical activity [126], signifying optical 
interactions of chiral molecules that differ, to some degree, for left- and right-handed opposite forms. In natural optical activity, chiral molecules of each kind interact differently with left- or right-handed circularly polarized light; i.e., a righthanded molecule will absorb left-handed light at a different rate than right-handed light, and this particular chiroptical process is known as circular dichroism. Other important forms include optical rotation and circular-differential scattering. The latter of these is also known as Rayleigh or Raman optical activity (ROA), depending on the type of scattering taking place. While Rayleigh optical activity has so far seen little application, ROA is an extremely important chiral spectroscopy, where it has been used to characterize the structures of important biomolecules such as nucleic acids, viruses, and even molecules as large as insulin [127-131]. ROA was an effect first predicted using SCT methods [132,133], with Barron going on to significantly develop the theory and pioneer the first experimental studies $[131,134]$. Although SCT was used in its prediction, the QED derivation of the ROA signal comes in an arguably more direct and straightforward manner, due to the way the theory is cast [5] (the situation is similar for circular dichroism too [135]). Importantly, however, both give equal results, though QED proved more amenable to a determination of the scattering-angle dependence of circular-differential scattering [136].

Among the most prominent successes of molecular QED has been its development of theory for RET-the ostensibly radiationless mechanism through which electronic excitation energy acquired by a donor molecule migrates to any acceptor with suitable spectral overlap [137-140]. This is a topic of wide relevance to a host of materials, including photosynthetic systems. The long-standing semiclassical treatment encapsulated in the well-known Förster theory is based on a nonretarded description of electromagnetic fields [141], which was shown to strictly fail to conform to the demands of causality $[142,143]$. By correctly describing the interaction between the transition moments of the donor and acceptor in terms of virtual photon propagation, it emerges from QED analysis that the short- and long-range mechanisms previously considered radiationless and radiative, respectively, are simply two asymptotic limits of a unified theory of energy transfer, fully accommodating both retardation and quantum uncertainty effects [123,124,144-148]. Inter alia, the QED analysis also showed that the two supposedly different mechanisms can never compete in determining the distance-dependent dynamics of energy transfer. The results, which have now become standard textbook material in the field of nano-optics [45], have found recent applications on energy transfer in engineered nanoscale structures $[149,150]$.

Even when SCT gives almost identical results to the QED picture, such as in multiphoton absorption [151-153], it is of course true that the former cannot adequately capture the coherence and nonclassical properties of input laser light, which become important through the $n$-photon coherence factor $g^{(n)} \neq 1[6]$. Quantum electrodynamical studies have also identified a more general nonlocalized mechanism for parametric nonlinear phenomena $[88,154]$. In the case of spontaneous parametric downconversion, the output can accommodate entangled photon pairs produced at distinct and spatially separated optical centers in the nonlinear crystal. This is a result that potentially impacts fundamental resolution in those imaging techniques that use downconverted photon pairs [155], as well as supplying a design and fabrication criterion for an enhanced conversion rate in judiciously synthesized nanostructures. One very recent further instance of the distinct clarity afforded by QED methods is a means of characterizing the quadratic diamagnetic coupling to the electromagnetic field, an often neglected or unknown interaction term that is required to secure gauge-independent results [156,157]. Specifically, it is the multipolar formalism of QED that has enabled the diamagnetic contributions to nonlinear spectroscopy [158] and intermolecular interactions $[8,159,160]$ to be most readily extracted, along with the highlighting of their unique, dispersion-free characteristics.

\section{B. Original Predictions of QED}

The added physical insight afforded by casting optical interactions explicitly in terms of photons coupling to matter, as in QED, has led to numerous predictions of new phenomena. This category includes a surprisingly diverse range. Returning to chiral phenomena in relation to a previous example, one might consider induced circular dichroism (ICD), first predicted by Craig, Power, and Thirunamachandran over 40 years ago [161]. In ICD, a locally chiral environment, as may for example be produced in a chiral solvent, can essentially break the local symmetry of achiral solute molecules, giving rise to circular differential absorption by the latter species. The effect has developed significant applications; see, for example, Refs. [162-164]. Another phenomenon first predicted by QED is hyper-Rayleigh (and Raman) optical activity [165], which has only recently been observed experimentally $[86,166]$.

In another connection, QED methods have also blazed a distinctive trail in the search of mechanisms for all-optical control, all-optical switching, and optical transistor action. It was shown that a range of novel optically nonlinear effects can arise in conventional processes such as absorption, emission, and energy transfer, subjected to the simultaneous throughput of an off-resonant laser beam that itself emerges unchanged [167173]. The laser-assisted (optically controlled) effect on RET, for example, displays a fundamental mechanism closely related to the experimentally well-established phenomenon of optical binding. In a sense, the off-resonant beam here acts as an optical catalyst, conferring a new dimension of optical nonlinearity upon photoactive materials. The field, now ripe for technical development, has been surveyed in a recent review [174].

Also in the sphere of nonlinear optics, two-photon fluorescence RET involves the transfer of energy resulting from the absorption of two photons by a molecule acting as donor. This allows for the application of fluorescence RET (FRET) using low-energy incident light that minimizes photodestruction of living tissues. Therefore, biological uses, such as photodynamic therapy and bioimaging, number prominently among the experimental applications that followed, soon after the initial QED-based prediction [175]; see, for example, Refs. [176187]. A more exotic nonlinear effect discovered using QED is SHG mediated by six-wave mixing $[188,189]$, which affords coherent second-harmonic production in isotropic media where 
second-harmonic conversion is generally forbidden. QED theory has also predicted the existence of a six-wave mixing process with optical vortices [190]. Both of these six-wave mixing phenomena have been vindicated by subsequent experimental evidence [191,192].

Short-range cooperative effects between molecules were also discovered using QED, whereby a mutual pairwise interaction between molecules leads to cooperative two-photon absorption (also termed mean-frequency absorption) [193-195]. This mechanism results in entirely new features in the absorption spectrum, allowing for transitions that are forbidden without any such mutual interaction between molecules. Here, the effect was subsequently experimentally observed in matrixisolated solids, liquids, gases, and molecular beams [196-202]. Attention has recently been drawn to the connection between providing accurate descriptions of short-range interactions and making due allowance for causality, the latter emerging again, naturally, through the retarded field formulation that is intrinsic to QED [203]. Another perspective recently given by Li et al. [204], re-emphasizing the issue of spontaneous emission in the QED and SCT treatments of theory, has focused on important implications for aspects of chemical computation.

While the spontaneous emission of a photon has been brought up repeatedly as a canonical example to highlight one essential difference between SCT and QED, it still has contemporary relevance. For example, the QED theory of interatomic Coulombic decay has recently been developed [205] —a phenomenon in which an outer electron of an initially-ionized atom relaxes, spontaneously, into the lower-lying vacancy within its inner electron shell, and the resultant excess energy is transferred to a second atom [206]. Taking account of the retardation effects, it has been shown that QED predictions of interatomic Coulombic decay generate rates that may be orders of magnitudes higher than those expected on the basis of other theories.

In eliciting the physics for the majority of applications discussed above, it has generally proved sufficient to use plane-wave representations of the quantum radiation modes, together with their polarization properties. Nonetheless, the same QED methods are highly amenable to application in the sphere of structured light [207], leading, for example, to the discovery that it is possible to deliver vortex photons by direct emission from suitably excited arrays [208-210] and so paving the way for a range of technical developments and implementations [211-216]. Whether the optical orbital angular momentum (OAM) of structured light could be transferred to the internal electronic degrees of freedom of an atom or molecule has been a well-debated topic, with highly significant implications in spectroscopy [217]. The first attempt at tackling this question utilized QED methods and determined that, for dipole transitions, the OAM of light is transferred to the mechanical motion of particles only; the lowest-order interaction required for OAM to be transferred to an electron is through E2 transitions [218]. While certain other non-QED analyses argued for OAM transferral in the dipole approximation, this failed to be observed in experiments [219,220]. Very recently, the QED theory was experimentally vindicated in the observation of modified selection rules for absorption of OAM light in which two units of angular momentum, $2 \hbar$, were transferred to the

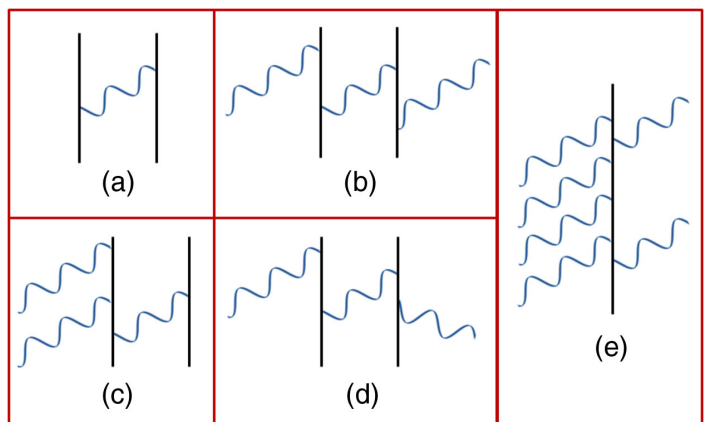

Fig. 6. Representative Feynman diagrams (in each case one of the full set of distinct time-orderings) depicting the QED mechanisms of (a) RET; (b) optically controlled RET (for all-optical switching); (c) two-photon fluorescence RET; (d) cooperative two-photon absorption; and (e) six-wave mixing. In all but (e), two centers represented as vertical lines are connected by a wavy line denoting virtual photon coupling: all other wavy lines denote real photons.

valence electron of a ${ }^{40} \mathrm{Ca}^{+}$ion through an $\mathrm{E} 2$ transition-one quantum of angular momentum from the spin angular momentum, and one from the OAM [221]. Alongside this recent accomplishment, there has also been a proof, again via QED, that the optical OAM of twisted light can produce chiroptical phenomena dependent on the direction of an optical vortex twist in both chiral and achiral matter [37,38,222-225]. With these successes - some illustrated in Fig. 6-and more, QED continues to make ongoing advances in the present day.

\section{CONCLUSION}

The legitimacy and requirement of the photon concept in optics is never in doubt, and when dealing with light-matter interactions at the atomic and molecular level, QED is the only theory in which the concept of the photon is completely valid. All we ever learn about light is through such interactions with matter: indeed our universe - matter and radiation-are unequivocally subject to quantum laws. There is a forceful logic, therefore, that QED ought always to be the default theory of choice. Nonetheless, it can be acknowledged that SCT is still more prevalent, and it is considered by many researchers to be easier to understand and deploy. As we have seen, this theory represents the electromagnetic radiation as a classical wave, but often it must still bolt on the additional requirement that light does indeed convey its energy in the form of quanta.

To reflect a broader context for the relative applicability of QED and semiclassical theories, it is worth pointing out a substantial difference in operation beyond the usual sphere of optics when concerning the dynamic modeling of material systems. It is true that, in certain situations, SCT can provide almost identical results as QED and, in many-body computational simulations, SCT may be preferable due to the much lower number of operations required. In this respect, there are two main reasons why SCT can be favored. In quantum theory, composite systems scale as the product of the dimensions of their individual Hilbert spaces, constructed through a tensor product [226]. By way of contrast, the corresponding number of dimensions in classical mechanics is a sum of the separate Hilbert space dimensions. Second, the time evolution of a quantum system 
requires evolving the unitary operator with the Hamiltonian as a complex exponential. Although it is possible to numerically evolve wave functions, commonly by expanding the unitary operator in a power series, the implementation dramatically scales upwards in computational time with every increase in order of perturbation. A common avoidance strategy is to transform to Hamilton's equations using semiclassical assumptions, and then treat the time-dependence classically.

As we have seen, a major downfall of SCT is its failure to correctly predict spontaneous emission. This phenomenon is not an intrinsic property of the material alone, but that of the material coupled to the quantum vacuum. It has been proven that, by incorporating boundaries to the quantum vacuum, this coupling can be manipulated, which leads to the field of cavity QED [227]. The latter is a distinctively separate field from the optical QED of interest to this review, but it does highlight another area of light-matter interactions that are specifically dependent upon the quantum nature of light. For further insight into the role that the vacuum field plays in spontaneous emission, alongside a historical overview of the differing physical interpretations, we refer the reader to the classic treatise by Milonni [24].

Producing theory that provides results to match previous or future empirical experimental analysis is, of course, the foremost criterion when judging the merit of any theory. To this end, it is clear that, with its broad compass in the physical sciences, QED has next to no competition. A secondary criterion is the level of physical insight that the theory allows, which has a distinct role to play in the predictive capacity of the ensuing theory. This deeper physical insight may also allow for the possibility of hitherto unrecognized optical phenomena to be conceptualized and visualized. In this respect, another distinct advantage of QED over SCT is the simplicity (though fully subject to quantum rigor) afforded through all light-matter interactions being cast through photon creation and annihilation events. Of course, what is most important from the technical viewpoint is the ability to recognize which framework is best suited for the problem at hand. This is why QED has furnished the underlying framework for a vast range of newly predicted and realized optical phenomena. Indeed, one other very recent study has underscored the interpretive power of this formalism to elicit the complex interplay of light and matter in numerous experimentally observed effects, as well as providing a route to new experiments at the interface between quantum chemistry, nanoplasmonics, and quantum optics [228].

In conclusion, for the robust formulation of theory for lightmatter processes, it is evident that a QED formalism proves simplest and neatest to implement. This framework works for all known forms of optics and photonics, and provides accurate physical insights. A strong case can be made that QED should be the theory of choice, since SCT has proven to fail in certain cases, even though, in many other applications, SCT will often produce the same results as QED. In our opinion, the mixing of theories that is seen in the literature is unwarranted, and obfuscates an already complex subject. QED can secure a dependable foundation for any optical process in optics and photonics, affording complete confidence in the resulting predictions.

\section{APPENDIX A: LARGE QUANTUM NUMBER ARGUMENTS}

Arguments based on large numbers of photons approaching classical behavior often prove specious since, unless one moves into the ultrahigh field regime, photon densities are seldom as high as might be supposed. A brief excursion into the numbers proves illuminating. Simple calculations somewhat undermine a common defense of SCT on the grounds that, due to the large numbers of photons presented by almost all sources of radiation, the "correspondence principle" can be assumed to generally apply. Certainly, this principle has validity for most traditional, and even laser-based optics, if the optical interactions and effects of interest are of significantly larger scale than the associated wavelength. But many key aspects of modern photonics involve features that necessarily involve highly localized interactionsany phenomenon or property that concerns the excitation of individual molecules or localized optical centers-or indeed any optical response in a nanoscale material or metamaterial, structured on a subwavelength scale.

Consider the photon density - the number of photons per unit volume in any essentially collimated beam. It is simple to show that, for a beam of irradiance $I$, the instantaneous mean number of photons within the $3 \mathrm{D}$ span of a wavelength is given by $I \lambda / h c^{2}$, and that for a beam of $500 \mathrm{~nm}$ radiation to sustain an average of even one photon in a $(500 \mathrm{~nm})^{3}$ volume requires an intensity of around $1 \mathrm{GW} \mathrm{m}{ }^{-2}$. This is a level that one might find near the focus of a cw argon ion laser, for example. And, of course, the region within which a localized electronic transition occurs is typically much smaller.

Perhaps even clearer is a calculation of the mean interval $\tau$ between the passage of successive photons through a molecule in the condensed phase (though it is, of course, subject to huge statistical fluctuations); $\tau=h c / I V^{2 / 3} \lambda$. So for a molecule of "typical" condensed phase volume, say an amino acid of molecular volume $\sim 0.1 \mathrm{~nm}^{3}$, the same $1 \mathrm{GW} \mathrm{m}{ }^{-2}$ intensity of light would ensure a photon within any molecular volume only once every $\sim 2$ ns: the mean number of photons within this volume would be about $10^{-9}$. Even in the radiofrequency range- the province of nuclear magnetic resonance spectroscopy - the numbers do not justify a "large number" assumption: the number of photons at any instant within the region occupied by a pair of spin-coupled protons would typically be about $10^{-6}(\mathrm{rf} 60 \mathrm{MHz}$, power $30-40 \mathrm{~W}$, tube area $0.5 \mathrm{~cm}^{2}$, volume $\left.10^{-2} \mathrm{~nm}^{3}\right)$. So there is good reason to suppose that the interactions of light, even in such familiar contexts, are realistically those of individual photons, and should, therefore, be treated as such.

Funding. Leverhulme Trust (ECF-2019-398); Deutsche Forschungsgemeinschaft (IRTG 2079); EU Horizon 2020 Framework Programme (75430).

Acknowledgment. We are grateful to Dr. Garth Jones, especially for his interesting insights on many-body simulations. KAF would like to thank the Leverhulme Trust for funding him through a Leverhulme Early Career Fellowship. AS acknowledges the award of a Mercator Fellowship funded by the Deutsche Forschungsgemeinschaft through the IRTG 
2079/Cold Controlled Ensembles in Physics and Chemistry at the University of Freiburg. He also thanks the Freiburg Institute for Advanced Studies (FRIAS), University of Freiburg for the award of a Marie S Curie External Senior Fellowship under the EU Horizon 2020.

\section{REFERENCES}

1. G. Grynberg, A. Aspect, and C. Fabre, Introduction to Quantum Optics: From the Semi-Classical Approach to Quantized Light (Cambridge University, 2010).

2. E. A. Power, Introductory Quantum Electrodynamics (American Elsevier, 1965).

3. R. G. Woolley, "The electrodynamics of atoms and molecules," in Advances in Chemical Physics (Wiley, 1975), Vol. 33, pp. 153-233.

4. C. Cohen-Tannoudji, J. Dupont-Roc, and G. Grynberg, Photons and Atoms: Introduction to Quantum Electrodynamics (Wiley, 1989).

5. D. P. Craig and T. Thirunamachandran, Molecular Quantum Electrodynamics: An Introduction to Radiation-Molecule Interactions (Dover, 1998).

6. R. Loudon, The Quantum Theory of Light (Oxford University, 2000).

7. D. L. Andrews and P. Allcock, Optical Harmonics in Molecular Systems (Wiley, 2002).

8. A. Salam, Molecular Quantum Electrodynamics. Long-Range Intermolecular Interactions (Wiley, 2010).

9. D. L. Andrews, G. A. Jones, A. Salam, and R. G. Woolley, "Perspective: quantum Hamiltonians for optical interactions," J. Chem. Phys. 148, 040901 (2018).

10. D. L. Andrews and D. S. Bradshaw, Introduction to Photon Science and Technology (SPIE, 2018).

11. D. L. Andrews, "Physicality of the photon," J. Phys. Chem. Lett. 4, 3878-3884 (2013).

12. H. Eyring, J. Walter, and G. E. Kimball, Quantum Chemistry (Wiley, 1944).

13. L. I. Schiff, Quantum Mechanics (McGraw-Hill, 1955).

14. M. Sargent, M. O. Scully, and W. E. Lamb, Laser Physics (CRC Press, 1978).

15. P. N. Butcher and D. Cotter, The Elements of Nonlinear Optics (Cambridge University, 1990).

16. N. Bloembergen, Nonlinear Optics, 4th ed. (World Scientific, 1996).

17. R. W. Boyd, Nonlinear Optics, 3rd ed. (Academic, 2008).

18. G. Gabrielse, D. Hanneke, T. Kinoshita, M. Nio, and B. Odom, "New determination of the fine structure constant from the electron g-value and QED," Phys. Rev. Lett. 97, 030802 (2006).

19. P. Schwerdtfeger, L. F. Pašteka, A. Punnett, and P. Bowman, "Relativistic and quantum electrodynamic effects in superheavy elements," Nucl. Phys. A 944, 551-577 (2015).

20. X. Zheng, Y. R. Sun, J. J. Chen, W. Jiang, K. Pachucki, and S. M. Hu, "Laser spectroscopy of the fine-structure splitting in the $2^{3} P_{J}$ levels of ${ }^{4} \mathrm{He}$," Phys. Rev. Lett. 118, 063001 (2017).

21. M. D. Crisp and E. T. Jaynes, "Radiative effects in semiclassical theory," Phys. Rev. 179, 1253-1261 (1969).

22. C. R. Stroud and E. T. Jaynes, "Long-term solutions in semiclassical radiation theory," Phys. Rev. A 1, 106-121 (1970).

23. R. van den Doel and J. J. J. Kokkedee, "Comment on the radiative level shift in the neoclassical radiation theory," Phys. Rev. A 9, 14681469 (1974).

24. P. W. Milonni, The Quantum Vacuum: An Introduction to Quantum Electrodynamics (Academic, 1993).

25. J. Schwinger, Selected Papers on Quantum Electrodynamics (Dover, 1958).

26. P. W. Milonni, "Semiclassical and quantum-electrodynamical approaches in nonrelativistic radiation theory," Phys. Rep. 25, 1-81 (1976).

27. E. A. Power and S. Zienau, "Coulomb gauge in non-relativistic quantum electrodynamics and the shape of spectral lines," Philos. Trans. R. Soc. A 251, 427-454 (1959).

28. R. G. Woolley, "Molecular quantum electrodynamics," Proc. R. Soc. A 321, 557-572 (1971).
29. R. G. Woolley, "Power-Zienau-Woolley representations of nonrelativistic QED for atoms and molecules," Phys. Rev. Res. 2, 013206 (2020).

30. D. L. Andrews, "Quantum formulation for nanoscale optical and material chirality: symmetry issues, space and time parity, and observables," J. Opt. 20, 033003 (2018).

31. D. L. Andrews, "Symmetries, conserved properties, tensor representations, and irreducible forms in molecular quantum electrodynamics," Symmetry 10, 298 (2018).

32. D. L. Andrews, "Effects of intrinsic local fields on molecular fluorescence and energy transfer: dipole mechanisms and surface potentials," J. Phys. Chem. B 123, 5015-5023 (2019).

33. D. L. Andrews, "Two-group Raman optical-activity revisited," Faraday Discuss. 99, 375-382 (1994).

34. D. L. Andrews and N. P. Blake, "Quantum electrodynamic study of bimolecular scattering effects in Raman-spectroscopy," Phys. Rev. A 41, 2547-2565 (1990).

35. D. L. Andrews, "An accretive mechanism for blue-shifted fluorescence in strongly pumped systems: resonance energy transfer with Raman emission," J. Raman Spectrosc. 31, 791-796 (2000).

36. M. D. Williams, D. S. Bradshaw, and D. L. Andrews, "Raman scattering mediated by neighboring molecules," J. Chem. Phys. 144, 174304 (2016).

37. K. A. Forbes, "Raman optical activity using twisted photons," Phys. Rev. Lett. 122, 103201 (2019).

38. K. A. Forbes and A. Salam, "Kramers-Heisenberg dispersion formula for scattering of twisted light," Phys. Rev. A 100, 053413 (2019).

39. G. Juzeliūnas, "Molecular-radiation and molecule-molecule processes in condensed media: a microscopic QED theory," Chem. Phys. 198, 145-158 (1995).

40. G. Juzeliūnas, "Microscopic theory of quantization of radiation in molecular dielectrics: normal-mode representation of operators for local and averaged (macroscopic) fields," Phys. Rev. A 53, 3543-3558 (1996).

41. Y. R. Shen, The Principles of Nonlinear Optics (Wiley, 2002).

42. G. S. He, Nonlinear Optics and Photonics (Oxford University, 2014).

43. J. S. Ford, A. Salam, and G. A. Jones, "A quantum electrodynamics description of quantum coherence and damping in condensedphase energy transfer," J. Phys. Chem. Lett. 10, 5654-5661 (2019).

44. J. D. Jackson, Classical Electrodynamics (Wiley, 1998).

45. L. Novotny and B. Hecht, Principles of Nano-Optics (Cambridge University, 2006).

46. J. Weiner and F. Nunes, Light-Matter Interaction. Physics and Engineering at the Nanoscale (Oxford University, 2013).

47. A. Zangwill, Modern Electrodynamics (Cambridge University, 2013).

48. A. Salam, "On the effect of a radiation field in modifying the intermolecular interaction between two chiral molecules," J. Chem. Phys. 124, 014302 (2006)

49. D. L. Andrews and D. S. Bradshaw, "The role of virtual photons in nanoscale photonics," Ann. Phys. (Berlin) 526, 173-186 (2014).

50. K. A. Forbes and D. L. Andrews, "Chiral discrimination in optical binding," Phys. Rev. A 91, 053824 (2015).

51. D. L. Andrews, "Chirality in fluorescence and energy transfer," Methods Appl. Fluoresc. 7, 032001 (2019).

52. J. H. Shirley, "Solution of the Schrödinger equation with a Hamiltonian periodic in time," Phys. Rev. 138, B979-B987 (1965).

53. S. Guérin and H. R. Jauslin, "Control of quantum dynamics by laser pulses: adiabatic Floquet theory," in Advances in Chemical Physics (Wiley, 2003), Vol. 125, pp. 147-268.

54. G. Compagno, K. Dietz, and F. Persico, "QED theory of harmonic emission by a strongly driven atom," J. Phys. B 27, 4779-4815 (1994).

55. M. Šindelka, "Derivation of coupled Maxwell-Schrödinger equations describing matter-laser interaction from first principles of quantum electrodynamics," Phys. Rev. A 81, 033833 (2010).

56. E. T. Jaynes and F. W. Cummings, "Comparison of quantum and semiclassical radiation theories with application to the beam maser," Proc. IEEE 51, 89-109 (1963). 
57. A. F. Kockum, A. Miranowicz, S. De Liberato, S. Savasta, and F. Nori, "Ultrastrong coupling between light and matter," Nat. Rev. Phys. 1, 19-40 (2019).

58. C. H. Wilcox, Perturbation Theory and its Applications in Quantum Mechanics (Wiley/Chapman and Hall, 1966).

59. C. Cohen-Tannoudji, J. Dupont-Roc, and G. Grynberg, AtomPhoton Interactions: Basic Processes and Applications (Wiley, 1992), p. 59.

60. D. L. Andrews, S. Naguleswaran, and G. E. Stedman, "Phenomenological damping of nonlinear-optical response tensors," Phys. Rev. A 57, 4925-4929 (1998).

61. D. L. Andrews, L. C. Dávila Romero, and G. E. Stedman, "Polarizability and the resonance scattering of light: damping sign issues," Phys. Rev. A 67, 055801 (2003).

62. P. W. Milonni and R. W. Boyd, "Influence of radiative damping on the optical-frequency susceptibility," Phys. Rev. A 69, 023814 (2004).

63. P. R. Berman, R. W. Boyd, and P. W. Milonni, "Polarizability and the optical theorem for a two-level atom with radiative broadening," Phys. Rev. A 74, 053816 (2006).

64. P. W. Milonni, R. Loudon, P. R. Berman, and S. M. Barnett, "Linear polarizabilities of two- and three-level atoms," Phys. Rev. A 77, 043835 (2008).

65. D. S. Bradshaw and D. L. Andrews, "Interparticle interactions: energy potentials, energy transfer, and nanoscale mechanical motion in response to optical radiation," J. Phys. Chem. A 117, 75-82 (2013).

66. E. Fermi, Nuclear Physics (University of Chicago, 1950).

67. D. S. Bradshaw, K. A. Forbes, and D. L. Andrews, "Quantum field representation of photon-molecule interactions," Eur. J. Phys. 41, 025406 (2020).

68. P. L. Knight and P. W. Milonni, "The Rabi frequency in optical spectra," Phys. Rep. 66, 21-107 (1980).

69. M. A. Parker, Physics of Optoelectronics (CRC Press, 2005).

70. A. Salam, "Molecular quantum electrodynamics in the Heisenberg picture: a field theoretic viewpoint," Int. Rev. Phys. Chem. 27, 405-448 (2008).

71. E. A. Power and T. Thirunamachandran, "Quantum electrodynamics with nonrelativistic sources. V. Electromagnetic field correlations and intermolecular interactions between molecules in either ground or excited states," Phys. Rev. A 47, 2539-2551 (1993).

72. P. R. Berman, Introductory Quantum Mechanics (Springer, 2018), p. 557.

73. A. Salam, Non-Relativistic QED Theory of the van der Waals Dispersion Interaction (Springer, 2016).

74. R. P. Feynman, "Space-time approach to quantum electrodynamics," Phys. Rev. 76, 769-789 (1949).

75. R. D. Jenkins, D. L. Andrews, and L. C. Dávila Romero, "A new diagrammatic methodology for non-relativistic quantum electrodynamics," J. Phys. B 35, 445-468 (2002).

76. D. L. Andrews and T. Thirunamachandran, "On three-dimensional rotational averages," J. Chem. Phys. 67, 5026-5033 (1977).

77. D. L. Andrews and W. A. Ghoul, "Eighth rank isotropic tensors and rotational averages," J. Phys. A 14, 1281-1290 (1981).

78. G. Wagnière, "The evaluation of three-dimensional rotational averages," J. Chem. Phys. 76, 473-480 (1982).

79. D. H. Friese, M. T. P. Beerepoot, and K. Ruud, "Rotational averaging of multiphoton absorption cross sections," J. Chem. Phys. 141, 204103 (2014).

80. T. Begzjav and R. Nessler, "On three-dimensional rotational averages of odd-rank tensors," Phys. Scr. 94, 105504 (2019).

81. D. Marcuse, Principles of Quantum Electronics (Elsevier, 2012).

82. E. Hendrickx, K. Clays, and A. Persoons, "Hyper-Rayleigh scattering in isotropic solution," Acc. Chem. Res. 31, 675-683 (1998).

83. R. D. Pyatt and D. P. Shelton, "Hyper-Rayleigh scattering from $\mathrm{CH}_{4}$, $\mathrm{CD}_{4}, \mathrm{CF}_{4}$, and $\mathrm{CCl}_{4}$," J. Chem. Phys. 114, 9938-9946 (2001).

84. E. C. Hao, G. C. Schatz, R. C. Johnson, and J. T. Hupp, "HyperRayleigh scattering from silver nanoparticles," J. Chem. Phys. 117, 5963-5966 (2002).

85. E. Botek, M. Spassova, B. Champagne, I. Asselberghs, A. Persoons, and K. Clays, "Hyper-Rayleigh scattering of neutral and charged helicenes," Chem. Phys. Lett. 412, 274-279 (2005).
86. J. T. Collins, K. R. Rusimova, D. C. Hooper, H. H. Jeong, L. Ohnoutek, F. Pradaux-Caggiano, T. Verbiest, D. R. Carbery, P. Fischer, and V. K. Valev, "First observation of optical activity in hyper-Rayleigh scattering," Phys. Rev. X 9, 011024 (2019).

87. M. D. Williams, J. S. Ford, and D. L. Andrews, "Hyper-Rayleigh scattering in centrosymmetric systems," J. Chem. Phys. 143, 124301 (2015).

88. K. A. Forbes, J. S. Ford, G. A. Jones, and D. L. Andrews, "Quantum delocalization in photon-pair generation," Phys. Rev. A 96, 023850 (2017).

89. D. L. Andrews and M. J. Harlow, "Phased and Boltzmann-weighted rotational averages," Phys. Rev. A 29, 2796-2806 (1984).

90. A. M. Stewart, "Why semiclassical electrodynamics is not gauge invariant," J. Phys. A 33, 9165-9175 (2000).

91. R. G. Woolley, "Charged particles, gauge invariance, and molecular electrodynamics," Int. J. Quant. Chem. 74, 531-545 (1999).

92. R. G. Woolley, "Gauge invariance in non-relativistic electrodynamics," Proc. R. Soc. A 456, 1803-1819 (2000).

93. J. F. Ward, "Calculation of nonlinear optical susceptibilities using diagrammatic perturbation theory," Rev. Mod. Phys. 37, 1-18 (1965).

94. D. L. Andrews, "A simple statistical treatment of multiphoton absorption," Am. J. Phys. 53, 1001-1002 (1985).

95. L. C. Dávila Romero, S. Naguleswaran, G. E. Stedman, and D. L. Andrews, "Electro-optic response in isotropic media," Nonlinear Opt. 23, 191-201 (2000).

96. D. L. Andrews, "Harmonic generation in free molecules," J. Phys. B 13, 4091-4099 (1980).

97. D. L. Andrews and N. P. Blake, "Forbidden nature of multipolar contributions to second-harmonic generation in isotropic fluids," Phys. Rev. A 38, 3113-3115 (1988).

98. D. S. Bradshaw and D. L. Andrews, "Quantum channels in nonlinear optical processes," J. Nonlinear Opt. Phys. Mater. 18, 285-299 (2009).

99. D. L. Andrews and D. S. Bradshaw, "A photonic basis for deriving nonlinear optical response," Eur. J. Phys. 30, 239-251 (2009).

100. D. L. Andrews and W. J. Meath, "On the role of permanent dipoles in second-harmonic generation," J. Phys. B 26, 4633-4641 (1993).

101. A. J. Moad and G. J. Simpson, "A unified treatment of selection rules and symmetry relations for sum-frequency and second harmonic spectroscopies," J. Phys. Chem. B 108, 3548-3562 (2004).

102. N. Bloembergen, R. K. Chang, S. S. Jha, and C. H. Lee, "Optical second-harmonic generation in reflection from media with inversion symmetry," Phys. Rev. 174, 813-822 (1968).

103. D. S. Bradshaw and D. L. Andrews, "Optically induced forces and torques: interactions between nanoparticles in a laser beam," Phys. Rev. A 72, 033816 (2005)

104. A. Salam, "Molecular quantum electrodynamics of radiationinduced intermolecular forces," in Advances in Quantum Chemistry, J. R. Sabin and E. Brandas, eds. (Academic, 2011), pp. 1-34.

105. Y. Harada and T. Asakura, "Radiation forces on a dielectric sphere in the Rayleigh scattering regime," Opt. Commun. 124, 529-541 (1996).

106. N. Malagnino, G. Pesce, A. Sasso, and E. Arimondo, "Measurements of trapping efficiency and stiffness in optical tweezers," Opt. Commun. 214, 15-24 (2002).

107. K. Dholakia, P. Reece, and M. Gu, "Optical micromanipulation," Chem. Soc. Rev. 37, 42-55 (2008).

108. G. Gouesbet, "Generalized Lorenz-Mie theories, the third decade: a perspective," J. Quant. Spectrosc. Radiat. Transfer 110, 1223-1238 (2009).

109. G. Gouesbet, "Latest achievements in generalized Lorenz-Mie theories: a commented reference database," Ann. Phys. (Berlin) 526, 461-489 (2014).

110. D. L. Andrews and D. S. Bradshaw, Optical Nanomanipulation (Morgan \& Claypool, 2016)

111. H. B. G. Casimir and D. Polder, "The influence of retardation on the London-van der Waals forces," Phys. Rev. 73, 360-372 (1948).

112. T. Čižmár, L. C. Dávila Romero, K. Dholakia, and D. L. Andrews, "Multiple optical trapping and binding: new routes to self-assembly," J. Phys. B 43, 102001 (2010). 
113. K. Dholakia and P. Zemanek, "Gripped by light: optical binding," Rev. Mod. Phys. 82, 1767-1791 (2010).

114. R. W. Bowman and M. J. Padgett, "Optical trapping and binding," Rep. Prog. Phys. 76, 026401 (2013).

115. L. C. Dávila Romero, J. Rodríguez, and D. L. Andrews, "Electrodynamic mechanism and array stability in optical binding," Opt. Commun. 281, 865-870 (2008).

116. J. Rodríguez, L. C. Dávila Romero, and D. L. Andrews, "Optical binding in nanoparticle assembly: potential energy landscapes," Phys. Rev. A 78, 043805 (2008).

117. Z. Yan, S. K. Gray, and N. F. Scherer, "Potential energy surfaces and reaction pathways for light-mediated self-organization of metal nanoparticle clusters," Nat. Commun. 5, 3751 (2014).

118. T. Thirunamachandran, "Intermolecular interactions in the presence of an intense radiation field," Mol. Phys. 40, 393-399 (1980).

119. M. M. Burns, J.-M. Fournier, and J. A. Golovchenko, "Optical binding," Phys. Rev. Lett. 63, 1233-1236 (1989).

120. D. S. Bradshaw, K. A. Forbes, J. M. Leeder, and D. L. Andrews, "Chirality in optical trapping and optical binding," Photonics 2, 483-497 (2015)

121. D. S. Bradshaw and D. L. Andrews, "Electromagnetic trapping of chiral molecules: orientational effects of the irradiating beam," J. Opt. Soc. Am. B 32, B25-B31 (2015).

122. K. A. Forbes, D. S. Bradshaw, and D. L. Andrews, "Optical binding of nanoparticles," Nanophotonics 9, 1-17 (2020).

123. G. J. Daniels, R. D. Jenkins, D. S. Bradshaw, and D. L. Andrews, "Resonance energy transfer: the unified theory revisited," J. Chem. Phys. 119, 2264-2274 (2003).

124. D. L. Andrews and D. S. Bradshaw, "Virtual photons, dipole fields and energy transfer: a quantum electrodynamical approach," Eur. J. Phys. 25, 845-858 (2004).

125. W. Heitler, The Quantum Theory of Radiation (Dover, 1984).

126. L. D. Barron, Molecular Light Scattering and Optical Activity (Cambridge University, 2009).

127. L. D. Barron, L. Hecht, E. W. Blanch, and A. F. Bell, "Solution structure and dynamics of biomolecules from Raman optical activity," Prog. Biophys. Mol. Biol. 73, 1-49 (2000).

128. L. D. Barron, E. W. Blanch, I. H. McColl, C. D. Syme, L. Hecht, and K. Nielsen, "Structure and behaviour of proteins, nucleic acids and viruses from vibrational Raman optical activity," J. Spectrosc. 17, 101-126 (2003).

129. L. D. Barron, L. Hecht, I. H. McColl, and E. W. Blanch, "Raman optical activity comes of age," Mol. Phys. 102, 731-744 (2004).

130. S. Yamamoto, J. Kaminský, and P. Bouř, "Structure and vibrational motion of insulin from Raman optical activity spectra," Anal. Chem. 84, 2440-2451 (2012).

131. L. D. Barron, "The development of biomolecular Raman optical activity spectroscopy," Biomed. Spectrosc. Imag. 4, 223-253 (2015).

132. L. D. Barron and A. D. Buckingham, "Rayleigh and Raman scattering from optically active molecules," Mol. Phys. 20, 1111-1119 (1971).

133. L. D. Barron and A. D. Buckingham, "Rayleigh and Raman optical activity," Annu. Rev. Phys. Chem. 26, 381-396 (1975).

134. P. I. Haris, "Laurence Barron: the founding father of Raman optical activity," Biomed. Spectrosc. Imag. 4, 219-222 (2015).

135. E. A. Power and T. Thirunamachandran, "Circular dichroism: a general theory based on quantum electrodynamics," J. Chem. Phys. 60 3695-3701 (1974).

136. D. L. Andrews, "Rayleigh and Raman optical activity: an analysis of the dependence on scattering angle," J. Chem. Phys. $\mathbf{7 2}$, 4141-4144 (1980).

137. D. L. Andrews and A. A. Demidov, Resonance Energy Transfer (Wiley, 1999)

138. V. May and O. Kühn, Charge and Energy Transfer Dynamics in Molecular Systems (Wiley, 2008).

139. D. L. Andrews and D. S. Bradshaw, "Resonance energy transfer," in Encyclopedia of Applied Specteoscopy, D. L. Andrews, eds. (Wiley, 2009), pp. 533-554.

140. G. A. Jones and D. S. Bradshaw, "Resonance energy transfer: from fundamental theory to recent applications," Front. Phys. 7, 100 (2019).
141. B. W. van der Meer, G. Coker, and S. Y. S. Chen, Resonance Energy Transfer: Theory and Data (VCH, 1994).

142. J. S. Avery, "Resonance energy transfer and spontaneous photon emission," Proc. Phys. Soc. London 88, 1-8 (1966).

143. L. Gomberoff and E. A. Power, "The resonance transfer of excitation," Proc. Phys. Soc. London 88, 281-284 (1966).

144. D. L. Andrews, "A unified theory of radiative and radiationless molecular energy transfer," Chem. Phys. 135, 195-201 (1989).

145. A. Salam, "Resonant transfer of excitation between two molecules using Maxwell fields," J. Chem. Phys. 122, 044113 (2005).

146. J. E. Frost and G. A. Jones, "A quantum dynamical comparison of the electronic couplings derived from quantum electrodynamics and Förster theory: application to 2D molecular aggregates," New J. Phys. 16, 113067 (2014).

147. R. Grinter and G. A. Jones, "Resonance energy transfer: the unified theory via vector spherical harmonics," J. Chem. Phys. 145, 074107 (2016).

148. A. Salam, "The unified theory of resonance energy transfer according to molecular quantum electrodynamics," Atoms 6, 56 (2018).

149. D. Weeraddana, M. Premaratne, S. D. Gunapala, and D. L. Andrews, "Quantum electrodynamical theory of high-efficiency excitation energy transfer in laser-driven nanostructure systems," Phys. Rev. B 94, 085133 (2016).

150. D. Weeraddana, M. Premaratne, S. D. Gunapala, and D. L. Andrews, "Controlling resonance energy transfer in nanostructure emitters by positioning near a mirror," J. Chem. Phys. 147, 074117 (2017).

151. W. J. Meath and E. A. Power, "On the effects of diagonal dipole matrix elements in multi-photon resonance profiles using two-level systems as models," Mol. Phys. 51, 585-600 (1984).

152. W. J. Meath and E. A. Power, "On the importance of permanent moments in multiphoton absorption using perturbation theory," J. Phys. B 17, 763-781 (1984).

153. W. J. Meath and E. A. Power, "On the interaction of elliptically polarized light with molecules: the effects of both permanent and transition multipole moments on multiphoton absorption and chiroptical effects," J. Mod. Opt. 36, 977-1002 (1989).

154. K. A. Forbes, J. S. Ford, and D. L. Andrews, "Nonlocalized generation of correlated photon pairs in degenerate down-conversion," Phys. Rev. Lett. 118, 133602 (2017).

155. P.-A. Moreau, E. Toninelli, T. Gregory, and M. J. Padgett, "Ghost imaging using optical correlations," Laser Photon. Rev. 12, 1700143 (2018).

156. K. A. Forbes, "Role of magnetic and diamagnetic interactions in molecular optics and scattering," Phys. Rev. A 97, 053832 (2018).

157. C. Schäfer, M. Ruggenthaler, V. Rokaj, and A. Rubio, "Relevance of the quadratic diamagnetic and self-polarization terms in cavity quantum electrodynamics," ACS Photon. (in press).

158. K. A. Forbes, D. S. Bradshaw, and D. L. Andrews, "Identifying diamagnetic interactions in scattering and nonlinear optics," Phys. Rev. A 94, 033837 (2016).

159. A. Salam, "On the contribution of the diamagnetic coupling term to the two-body retarded dispersion interaction," J. Phys. B 33, 2181$2193(2000)$.

160. S. Y. Buhmann, H. Safari, S. Scheel, and A. Salam, "Body-assisted dispersion potentials of diamagnetic atoms," Phys. Rev. A 87, 012507 (2013).

161. D. P. Craig, E. A. Power, and T. Thirunamachandran, "The dynamic terms in induced circular dichroism," Proc. R. Soc. A 348, 19-38 (1976).

162. S. Allenmark, "Induced circular dichroism by chiral molecular interaction," Chirality 15, 409-422 (2003).

163. D. Tedesco and C. Bertucci, "Induced circular dichroism as a tool to investigate the binding of drugs to carrier proteins: classic approaches and new trends," J. Pharm. Biomed. Anal. 113, 34-42 (2015).

164. A. Hong, C. J. Moon, H. Jang, A. Min, M. Y. Choi, J. Heo, and N. J. Kim, "Isomer-specific induced circular dichroism spectroscopy of jet-cooled phenol complexes with (-)-methyl I-lactate," J. Phys. Chem. Lett. 9, 476-480 (2018).

165. D. L. Andrews and T. Thirunamachandran, "Hyper-Raman scattering by chiral molecules," J. Chem. Phys. 70, 1027-1030 (1979). 
166. D. Verreault, K. Moreno, É. Merlet, F. Adamietz, B. Kauffmann, Y. Ferrand, C. Olivier, and V. Rodriguez, "Hyper-Rayleigh scattering as a new chiroptical method: uncovering the nonlinear optical activity of aromatic oligoamide foldamers," J. Am. Chem. Soc. 142, 257-263 (2020).

167. D. S. Bradshaw and D. L. Andrews, "Optically controlled resonance energy transfer: mechanism and configuration for all-optical switching," J. Chem. Phys. 128, 144506 (2008).

168. D. S. Bradshaw and D. L. Andrews, "The control of near-field optics: imposing direction through coupling with off-resonant laser light," Appl. Phys. B 93, 13-20 (2008).

169. D. S. Bradshaw and D. L. Andrews, "Mechanism for optical enhancement and suppression of fluorescence," J. Phys. Chem. A 113, 6537-6539 (2009)

170. D. L. Andrews and D. S. Bradshaw, "Off-resonant activation of optical emission," Opt. Commun. 283, 4365-4367 (2010).

171. D. S. Bradshaw and D. L. Andrews, "All-optical control of molecular fluorescence," Phys. Rev. A 81, 013424 (2010).

172. D. S. Bradshaw and D. L. Andrews, "All-optical switching between quantum dot nanoarrays," Superlatt. Microstruct. 47, 308-313 (2010).

173. J. M. Leeder, D. S. Bradshaw, and D. L. Andrews, "Laser-controlled fluorescence in two-level systems," J. Phys. Chem. B 115, 5227-5233 (2011).

174. D. S. Bradshaw, K. A. Forbes, and D. L. Andrews, "Off-resonance control and all-optical switching: expanded dimensions in nonlinear optics," Appl. Sci. 9, 4252 (2019).

175. P. Allcock and D. L. Andrews, "Two-photon fluorescence: resonance energy transfer," J. Chem. Phys. 108, 3089-3095 (1998).

176. W. R. Dichtel, J. M. Serin, C. Edder, J. M. J. Fréchet, M. Matuszewski, L.-S. Tan, T. Y. Ohulchanskyy, and P. N. Prasad, "Singlet oxygen generation via two-photon excited FRET," J. Am. Chem. Soc. 126, 5380-5381 (2004).

177. G. F. White, K. L. Litvinenko, S. R. Meech, D. L. Andrews, and A. J. Thomson, "Multiphoton-excited luminescence of a lanthanide ion in a protein complex: $\mathrm{Tb}^{3+}$ bound to transferrin," Photochem. Photobiol. Sci. 3, 47-55 (2004).

178. M. A. Oar, J. M. Serin, W. R. Dichtel, J. M. J. Fréchet, T. Y. Ohulchanskyy, and P. N. Prasad, "Photosensitization of singlet oxygen via two-photon-excited fluorescence resonance energy transfer in a water-soluble dendrimer," Chem. Mater. 17, 2267-2275 (2005).

179. N. Tian and Q.-H. Xu, "Enhanced two-photon excitation fluorescence by fluorescence resonance energy transfer using conjugated polymers," Adv. Mater. 19, 1988-1991 (2007).

180. S. Kim, H. Huang, H. E. Pudavar, Y. Cui, and P. N. Prasad, "Intraparticle energy transfer and fluorescence photoconversion in nanoparticles: an optical highlighter nanoprobe for two-photon bioimaging," Chem. Mater. 19, 5650-5656 (2007).

181. S.-H. Cheng, C.-C. Hsieh, N.-T. Chen, C.-H. Chu, C.-M. Huang, P.-T. Chou, F.-G. Tseng, C.-S. Yang, C.-Y. Mou, and L.-W. Lo, "Welldefined mesoporous nanostructure modulates three-dimensional interface energy transfer for two-photon activated photodynamic therapy," Nano Today 6(6), 552-563 (2011).

182. E. J. Ngen, L. Xiao, P. Rajaputra, X. Yan, and Y. You, "Enhanced singlet oxygen generation from a porphyrin-rhodamine $B$ dyad by twophoton excitation through resonance energy transfer," Photochem. Photobiol. 89, 841-848 (2013).

183. N.-T. Chen, K.-C. Tang, M.-F. Chung, S.-H. Cheng, C.-M. Huang, C.-H. Chu, P.-T. Chou, J. S. Souris, C.-T. Chen, C.-Y. Mou, and L.-W. Lo, "Enhanced plasmonic resonance energy transfer in mesoporous silica-encased gold nanorod for two-photon-activated photodynamic therapy," Theranostics 4, 798-807 (2014).

184. Y. Acosta, Q. Zhang, A. Rahaman, H. Ouellet, C. Xiao, J. Sun, and C. Li, "Imaging cytosolic translocation of mycobacteria with twophoton fluorescence resonance energy transfer microscopy," Biomed. Opt. Express 5, 3990-4001 (2014).

185. T. He, R. Chen, Z. B. Lim, D. Rajwar, L. Ma, Y. Wang, Y. Gao, A. C. Grimsdale, and H. Sun, "Efficient energy transfer under twophoton excitation in a 3D, supramolecular, Zn(II)-coordinated, self-assembled organic network," Adv. Opt. Mater. 2, 40-47 (2014).
186. S. Drozdek, J. Szeremeta, L. Lamch, M. Nyk, M. Samoc, and K. A. Wilk, "Two-photon induced fluorescence energy transfer in polymeric nanocapsules containing $\mathrm{CdSe}_{x} \mathrm{~S}_{1-x} / \mathrm{ZnS}$ core/shell quantum dots and zinc(II) phthalocyanine," J. Phys. Chem. C 120 , 15460-15470 (2016).

187. M. Zhu, J. Zhang, Y. Zhou, P. Xing, L. Gong, C. Su, D. Qi, H. Du, Y. Bian, and J. Jiang, "Two-photon excited FRET dyads for lysosometargeted imaging and photodynamic therapy," Inorg. Chem. 57, 11537-11542 (2018).

188. P. Allcock and D. L. Andrews, "Six-wave mixing: secular resonances in a higher-order mechanism for second-harmonic generation," J. Phys. B 30, 3731-3742 (1997).

189. I. D. Hands, S. Lin, S. R. Meech, and D. L. Andrews, "A quantum electrodynamical treatment of second harmonic generation through phase conjugate six-wave mixing: polarization analysis," J. Chem. Phys. 109, 10580-10586 (1998).

190. M. M. Coles, M. D. Williams, and D. L. Andrews, "Second harmonic generation in isotropic media: six-wave mixing of optical vortices," Opt. Express 21, 12783-12789 (2013).

191. K. D. Moll, D. Homoelle, A. L. Gaeta, and R. W. Boyd, "Conical harmonic generation in isotropic materials," Phys. Rev. Lett. 88, 153901 (2002).

192. D. Zhang, X. Liu, L. Yang, X. Li, Z. Zhang, and Y. Zhang, "Modulated vortex six-wave mixing," Opt. Lett. 42, 3097-3100 (2017).

193. D. L. Andrews and M. J. Harlow, "Cooperative two-photon absorption," J. Chem. Phys. 78, 1088-1094 (1983).

194. D. L. Andrews and M. J. Harlow, "Cooperative two-photon absorption. II," J. Chem. Phys. 80, 4753-4760 (1984).

195. Z. Zheng, P. L. Saldanha, J. R. Rios Leite, and C. Fabre, "Twophoton-two-atom excitation by correlated light states," Phys. Rev. A 88, 033822 (2013).

196. J. K. Ku, G. Inoue, and D. W. Setser, "Two-photon laser-assisted reaction with xenon/molecular chlorine to form excited xenon chloride $\left(\mathrm{XeCl}^{*}\right)$ and with xenon/iodine chloride $(\mathrm{ICl})$ to form excited xenon chloride $\left(\mathrm{XeCl}^{*}\right)$ and excited xenon iodide (Xel*)," J. Phys. Chem. 87, 2989-2993 (1983).

197. M. E. Fajardo and V. A. Apkarian, "Cooperative photoabsorption induced charge transfer reaction dynamics in rare gas solids. I. Photodynamics of localized xenon chloride exciplexes," J. Chem. Phys. 85, 5660-5681 (1986).

198. M. Boivineau, J. Le Calvé, M. C. Castex, and C. Jouvet, "Formation of the $\mathrm{XeBr}^{*}$ excimer by double optical excitation of the $\mathrm{Xe}-\mathrm{Br}_{2}$ van der Waals complex," J. Chem. Phys. 84, 4712-4713 (1986).

199. L. Wiedeman, M. E. Fajardo, and V. A. Apkarian, "Electronic relaxation of xenon chloride $\left(\mathrm{Xe}_{2}{ }^{+} \mathrm{Cl}^{-}\right)$in solid and liquid xenon," J. Phys. Chem. 92, 342-346 (1988).

200. D. L. Andrews and K. P. Hopkins, "Synergistic effects in twophoton absorption: the quantum electrodynamics of bimolecular mean-frequency absorption," Adv. Chem. Phys. 77, 39-102 (1990).

201. J. Kohel and J. W. Keto, "Energy disposal in the two-photon laserassisted reaction in xenon and chlorine gas mixtures," J. Chem. Phys. 113, 10551-10559 (2000).

202. E. Pedrozo-Peñafiel, R. R. Paiva, F. J. Vivanco, V. S. Bagnato, and K. M. Farias, "Two-photon cooperative absorption in colliding cold Na atoms," Phys. Rev. Lett. 108, 253004 (2012).

203. T. E. Li, H.-T. Chen, A. Nitzan, M. Sukharev, and J. E. Subotnik, "A necessary trade-off for semiclassical electrodynamics: accurate short-range Coulomb interactions versus the enforcement of causality?" J. Phys. Chem. Lett. 9, 5955-5961 (2018).

204. T. E. Li, H.-T. Chen, and J. E. Subotnik, "Comparison of different classical, semiclassical, and quantum treatments of light-matter interactions: understanding energy conservation," J. Chem. Theory Comput. 15, 1957-1973 (2019).

205. J. L. Hemmerich, R. Bennett, and S. Y. Buhmann, "The influence of retardation and dielectric environments on interatomic Coulombic decay," Nat. Commun. 9, 2934 (2018).

206. T. Jahnke, "Interatomic and intermolecular Coulombic decay: the coming of age story," J. Phys. B 48, 082001 (2015).

207. L. C. Dávila Romero, D. L. Andrews, and M. Babiker, "A quantum electrodynamics framework for the nonlinear optics of twisted beams," J. Opt. B 4, S66-S72 (2002). 
208. M. D. Williams, M. M. Coles, K. Saadi, D. S. Bradshaw, and D. L. Andrews, "Optical vortex generation from molecular chromophore arrays," Phys. Rev. Lett. 111, 153603 (2013).

209. M. M. Coles, M. D. Williams, K. Saadi, D. S. Bradshaw, and D. L. Andrews, "Chiral nanoemitter array: a launchpad for optical vortices," Laser Photon. Rev. 7, 1088-1092 (2013).

210. M. D. Williams, M. M. Coles, D. S. Bradshaw, and D. L. Andrews, "Direct generation of optical vortices," Phys. Rev. A 89, 033837 (2014).

211. R. Liu, D. B. Phillips, F. Li, M. D. Williams, D. L. Andrews, and M. J. Padgett, "Discrete emitters as a source of orbital angular momentum," J. Opt. 17, 045608 (2015).

212. M. Asano and T. Takahashi, "Analytical investigation of optical vortices emitted from a collectively polarized dipole array," Opt. Express 23, 27998-28011 (2015).

213. X. Zang and M. T. Lusk, "Twisted molecular excitons as mediators for changing the angular momentum of light," Phys. Rev. A 96, 013819 (2017).

214. A. Forbes, "Controlling light's helicity at the source: orbital angular momentum states from lasers," Philos. Trans. R. Soc. A 375 , 20150436 (2017).

215. A. Zannotti, F. Diebel, M. Boguslawski, and C. Denz, "Chiral light in helically twisted photonic lattices," Adv. Opt. Mater. 5, 1600629 (2017).

216. R. M. Kerber, J. M. Fitzgerald, S. S. Oh, D. E. Reiter, and O. Hess, "Orbital angular momentum dichroism in nanoantennas," Commun. Phys. 1, 87 (2018).

217. M. Babiker, D. L. Andrews, and V. E. Lembessis, "Atoms in complex twisted light," J. Opt. 21, 013001 (2019).

218. M. Babiker, C. R. Bennett, D. L. Andrews, and L. C. Dávila Romero, "Orbital angular momentum exchange in the interaction of twisted light with molecules," Phys. Rev. Lett. 89, 143601 (2002).
219. R. Mathevet, B. V. de Lesegno, L. Pruvost, and G. L. J. A. Rikken, "Negative experimental evidence for magneto-orbital dichroism," Opt. Express 21, 3941-3945 (2013).

220. F. Giammanco, A. Perona, P. Marsili, F. Conti, F. Fidecaro, S. Gozzini, and A. Lucchesini, "Influence of the photon orbital angular momentum on electric dipole transitions: negative experimental evidence," Opt. Lett. 42, 219-222 (2017).

221. C. T. Schmiegelow, J. Schulz, H. Kaufmann, T. Ruster, U. G. Poschinger, and F. Schmidt-Kaler, "Transfer of optical orbital angular momentum to a bound electron,” Nat. Commun. 7, 12998 (2016).

222. D. L. Andrews, L. C. D. Romero, and M. Babiker, "On optical vortex interactions with chiral matter," Opt. Commun. 237, 133-139 (2004).

223. K. A. Forbes and D. L. Andrews, "Optical orbital angular momentum: twisted light and chirality," Opt. Lett. 43, 435-438 (2018).

224. K. A. Forbes and D. L. Andrews, "Spin-orbit interactions and chiroptical effects engaging orbital angular momentum of twisted light in chiral and achiral media," Phys. Rev. A 99, 023837 (2019).

225. K. A. Forbes and D. L. Andrews, "Enhanced optical activity using the orbital angular momentum of structured light," Phys. Rev. Res. 1, 033080 (2019).

226. M. A. Nielsen and I. L. Chuang, Quantum Computation and Quantum Information, 10th anniversary eds. (Cambridge University, 2010).

227. H. Walther, B. T. H. Varcoe, B.-G. Englert, and T. Becker, "Cavity quantum electrodynamics," Rep. Prog. Phys. 69, 1325-1382 (2006).

228. J. Flick, D. M. Welakuh, M. Ruggenthaler, H. Appel, and A. Rubio, "Light-matter response in nonrelativistic quantum electrodynamics," ACS Photon. 6, 2757-2778 (2019). 